
Italique

\section{Italique}
Poésie italienne de la Renaissance

XI | 2008

Varia

\title{
La Predica d'Amore : note sulla parodia sacra tra Quattro e Cinquecento
}

\section{Matteo Largaiolli}

\section{OpenEdition}

\section{Journals}

\section{Edizione digitale}

URL: http://journals.openedition.org/italique/210

DOI: $10.4000 /$ italique.210

ISSN: 1663-4438

\section{Editore}

Librairie Droz

\section{Edizione cartacea}

Data di pubblicazione: 1 dicembre 2008

Paginazione: 53-89

ISBN: 978-2-600-01254-6

ISSN: 1423-3983

Notizia bibliografica digitale

Matteo Largaiolli, "La Predica d'Amore : note sulla parodia sacra tra Quattro e Cinquecento », Italique [Online], XI | 2008, online dal 01 février 2012, consultato il 21 avril 2019. URL : http://

journals.openedition.org/italique/210; DOI : 10.4000/italique.210

(C) Tous droits réservés 


\author{
Matteo Largaiolli \\ L A PREDICA D'AMORE \\ NOTE S ULLA PARODIA SACRA \\ TRA Q U A T R O E C I N Q E C E N T O
}




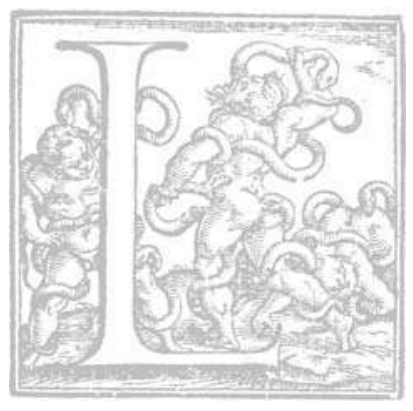

A Predica d'Amore è una forma di parodia sacra, diffusa tra Quattro e Cinquecento, che adotta la struttura del sermone sacro per comunicare contenuti erotici. Il quadro offerto dalle Prediche d'Amore è vario: ci sono prediche più e prediche meno letterarie, prediche in versi e prediche in prosa, ma la struttura portante, parodia del sermo modernus, è costante e permette di considerare le prediche come appartenenti a un unico genere.

Destinate al divertimento in occasioni festive, legate alle tradizioni del carnevale, sia nelle corti e negli ambienti colti, sia in ambienti piu popolari, le Prediche d'Amore sono state trasmesse da manoscritti e da stampe che riflettono questi due versanti: manoscritti miscellanei di diversa estrazione collettori di letteratura "popolaresca" o cortigiana, raccolte poetiche a stampa, stampe popolari. La definizione di un repertorio di Prediche d'Amore vuole restituire visibilità a questo genere e ricostruirne il corpus nelle sue articolazioni interne.

I. La storia della parodia sacra è secolare e ricca di sfaccettature: dalla parodia dei personaggi biblici della Cena Cypriani, alla cultura mediolatina attraverso $i$ Carmina Burana, fino al passaggio, tra XII e XIII secolo, al volgare, con un cambio di prospettiva non solo linguistica, ma anche culturale, ${ }^{ }$ la parodia dimostra una vitalità e una versatilità di cui è testimonianza la relativa facilità con cui può assumere ruoli diversi a seconda delle epoche e delle circostanze in cui viene esercitata: «la parodia del sacro [...] mutò radicalmente di funzione in età rinascimentale, quando fu adibita a scopo politico e di disputa teologicas. ${ }^{2}$

La parodia sacra si estende dagli esempi di parodia scritturale come la parodia del Vangelo (Evangelium secundum Marcam argentii, che si legge nei Carmina Burana, 2I5), alle parodie di testi agiografici, che si specializzarono nella ricostruzione della biografia di santi immaginari, quali san Nemo e sant'Invicem, o alle parodie degli inni cristiani (con un orientamento privilegiato verso il vino come $i$ carmina potatoria, o la donna e il gioco), alle parodie di preghiere (ad esempio $i$ Paternostri in chiave baccbica). Tutte forme di parodia che sembrano infine compattarsi nella parodia più complessa dell'azione liturgica, di cui sono noti un Officium lusorum che si legge nei Carmina Burana e una Missa potatorum edita da Novati. ${ }^{3}$

Con le Missae parodiche si fa strada anche la parodia del sermone, una parte dell'azione liturgica che non era sempre strettamente vincolata alla celebrazione della messa e che aveva acquisito nel corso del Medioevo caratteri 
di vivace teatralità. Una teatralità che si ritrova nelle Prediche parodiche in cui Carnevale annuncia dal pulpito le meraviglie del Paese di Cuccagna, o le speculari disgrazie della fame. Le Prediche di Carnevale sono attestate fino a tutto il Cinquecento: forme giocose da fiera, sono di solito lunghi testi sul metro della frottola di settenari. ${ }^{4}$ Accanto a questo genere popolare, ripetitivo, monotematico e letterariamente poco avvertito, si affermano, con una più ricca gamma di realizzazioni, le Prediche d'Amore, parodia non di un unico, definito, riconoscibile testo, ma di tutto un genere - la predica formalizzata dalle artes praedicandi nel modello del sermo modernus. ${ }^{5}$

2. I contatti tra la Predica d'Amore $e$ il mondo carnevalesco sono espliciti nei titoli di alcune Prediche, carnascialesche, come le Prediche di Pier Luigi di Francesco Magdoli, o da recitare nel periodo di carnevale: Baldacchini titola la sua Predica «sermo devotissimus in Carnisprevio toto orbe celebrato die habendus ad expergiscendos amentes ut amantes fiant». In ogni caso, è abbastanza evidente che se una delle destinazioni privilegiate era il Carnevale, la parodia può travalicare il momento strettamente prequaresimale: anche altre feste possono essere occasione di diffusione del materiale a stampa. Le Prediche di Magdoli, ad esempio, presentate esplicitamente in frontespizio come carnascialesche, sono state stampate il 25 gingno I524, ed è difficile pensare che si sia atteso il gennaio o il febbraio dell'anno successivo per metterle in commercio. Come per Croce «le moment de la publication n'est pas laissé au hasard. Il est choisi en fonction du calendrier des fêtes qui marquent des temps de rupture dans le déroulement de l'année de travail: décembre et janvier pour le Noël et le Nouvel An; en février, à l'occasion des longues réjouissances populaires du Carnaval; pour la fête de la Porchetta, le 24 août, jour de la Saint-Barthélémyy. ${ }^{6}$

Altre testimonianze coeve sul rapporto con il carnevale sono rare. Novati riporta un passo di una lettera in cui Girolamo Muzio narrava $i$ «divertimenti carnevaleschi che egli godeva con un'allegra brigata), dichiarandosi autore egli stesso di una predica parodica, quasi un esercizio leggero e piacevole: «ho apparecchiato una Predica d'amore da dover recitar la Giobbia grassa o la sera del carnevale, quando più piacerà a chi la haverà da ascoltare». ${ }^{7}$ Queste poche parole, inoltre, indicano una destinazione recitativa e spettacolare della Predica d'Amore, simile in questo alle prediche di Carnevale.

Il riferimento al carnevale non va inteso solo nei suoi risvolti popolari: le occasioni festive favorivano la composizione di testi destinati al diletto di società anche nei circoli colti dell'aristocrazia politica e letteraria quattrocinquecentesca. Sannazaro compone le sue farse per le festività del Carnevale alla corte di Napoli. ${ }^{8}$ La Predica de' XII eremiti, di fatto una Predica 
d'Amore, segna una precisa corrispondenza tra l'attività letteraria colta ispirata alla tradizione carnevalesca e la predica erotica, oltre che un autorevole possibile precedente per gli altri esempi di Predica. La scrittura di farse, intermezzi e gliommeri destinati alle feste è dettata da occasioni contingenti, ma è ormai accertato il loro sfondo letterario; per la loro composizione si può quindi pensare a «interventi operati da autori colti, desiderosi di esercitare il proprio gusto del popolaresco, su un genere popolare pre-esistentes:? carnevali di corte, quindi, non popolari.

La Predica di Sannazaro (in endecasillabi frottolati, come lo gliommero) si apre, come dovuto, con l'esposizione in epigrafe del thema (Nil durum in pulchra nobilitate cadit) e con una preghiera a Venere ( $v v \cdot I-I \sigma)$, con un accenno alla destinazione omiletica del testo ("io sempre in la mia vita t'ho servito, / et or che so' eremito in continenzia / predico tua potenzia in questa terra》, vv. II-I3). Il predicatore procede con lillustrazione del destino delle donne crudeli, destinate ad un futuro di solitudine in vecchiaia (con $i$ topoi dell'ubi sunt e del tempo che corrompe la bellezza). La conclusione non può che essere una: "Donne, non siate ingrati a' vostri amanti», ampliata ai vv. I26-I28: «Notate mie parole e siate umane, / non ve mostrate strane né crudeli 1 agli amanti fidelis.

La predica di Sannazaro stabilisce un contatto tra Predica d'Amore, farsa, letteratura del carnevale: «nella Predica [de' XII eremiti] e ne La giovane e la vecchia viene instaurato un rapporto con la poesia popolare toscana e con quella carnascialesca in particolares. ${ }^{\text {I0 }}$ Tra le tradizioni del carnevale rilette in ambito colto e condivise almeno in parte dalla Predica d'Amore è significativa la presenza del motivo delle Allegrezze d'Amore, parodia delle Allegrezze della Vergine, speculari ai di lei Sette dolori (a loro volta parodiati nei Sette dolori d'Amore). L'esempio più noto delle Allegrezze d'Amore, catalogo delle tappe di sedurione che conducono alla conquista della donna amata fino alla definitiva e più dolce «ultima allegrezza», sono le ottave (Deh, state a udire, giovane e donzelle) che Lorenzo de' Medici scrive collocandole nel tempo del carnevale: "Il poveretto è già condotto a tale, / che non ha con chi fare il carnasciale» (vv. 8I-82). Alle stanze di Lorenzo si accostano le ottave di Bartolomeo Cavassico ${ }^{11}$ e alcune terzine che si leggono nel codice Marciano It. IX 66, cc. 445r e sg., tra componimenti dell'Aretino. Alla stessa tradizione rimontano anche alcuni versi $(v v$. 58-74) della Quarta parte della Predica attribuita a Marco Rosiglia. Attivo alla corte urbinate di Guidubaldo di Montefeltro e Elisabetta Gonzaga, Rosiglia aveva di fronte le feste per il carnevale cortigiano degli anni iniziali del XVI secolo. Nel I504 Vincenzo Colli si dedica alla stesura di una commedia, perduta, ma di cui rimane testimonianza in una lettera di Emilia Pio a Isabella d'Este del s marzo I504. ${ }^{12}$ Nel 1507, Bembo propose le Stanze recitate per giuoco 
da lui e dal S. Ottaviano Fregoso, mascherati a guisa di due ambasciatori della dea Venere, mandati a Lisabetta Gonzaga duchessa d'Urbino e Madonna Emilia Pio. ${ }^{13}$ Anche a voler trascurare la perduta commedia del Calmeta, andrà ricordato che nel I 508 «sempre a Carnevale, sempre a Urbino, e ancora per Elisabetta, Baldassarre Castiglione e Cesare Gonzaga avevano messo in scena [...] l'egloga dialogata intitolata Tirsi», ${ }^{\mathrm{I}} e_{e}$ nel isiz la Calandria.

3.I. Tutte le Prediche d'Amore adottano come testo-modello la predica sacra, definita e regolata dalle artes praedicandi nel corso del XII e XIII secolo nella forma del sermo modernus, che pur tra spinte contrastanti resiste fino a tutto il Quattrocento e fino agli inizi del Cinquecento. Le artes praedicandi prescrivevano il commento di un thema, un versetto scelto di solito dalle letture della liturgia del giorno, che veniva analizzato in tutti i suoi significati attraverso, principalmente, due tecniche. Il thema era sottoposto, cioè, a un'operazione di divisio $e$ scisso in parti, di solito tre, o a un'operazione di distinctio, in cui una sola parola del versetto era commentata in tutto il suo spettro di significati (anche in questo caso, spesso, erano selezionati tre significati principali). Ciascuna parte in cui era diviso il thema o in cui si distingueva una sua parola diventava il nucleo di una sezione della predica, che nella maggior parte dei casi era costruita, quindi, in tre parti. ${ }^{\text {Is }}$

La struttura rigidamente definita, la relativa facilità di imitazione una volta assimilato lo schema di base, e il conseguente facile riconoscimento del gioco di imitazione, la presenza di parti (come la pregbiera iniziale) e di formule ripetitive (come le benedizioni finali), la presenza di inserti nobilitanti e immediatamente identificabili (come gli exempla e le auctoritates), e ancora gli eccessi anche istrionici dell'esecuzione concreta, la diffusione stessa del sermo modernus, l'autorità sacra di cui godeva, sono tutti elementi che concorrono a spiegare la facile acquisizione del modello da parodiare e la fortuna della parodia - e si aggiunga che il genere, con più secoli di storia alle spalle, stava ormai cominciando a declinare, rendendo in qualche modo più legittima l'ironia.

Le Prediche d'Amore ricalcano la struttura tipica del sermone sacro: posta l'assunzione di un thema, questo viene elaborato nelle partes della predica. La scelta dei themata $e$, naturalmente, condizionata dall'interesse principale dei predicatori d'Amore. Come la predica sacra deve ammaestrare in materia di fede e di dogmi, deve spingere all'operare virtuoso, deve dimostrare che il premio per il fedele è la gioia eterna, così, con un rispecchiamento parallelo costante, le Prediche d'Amore ammaestrano in materia d'amore (con questioni di vaga filosofia, ad esempio sulla natura di Amore o la sua origine, ma anche con consigli concreti, al modo di una ars amandi), spingono all'espe- 
rienza erotica (in termini esplicitamente sensuali: l'obiettivo di ogni fedele di Amore deve essere la soddisfazione del proprio desiderio erotico, e ogni donna sarà virtuosa se si concederà al suo amante), assicurano per quanti metteranno in pratica gli insegnamenti esposti una pace e una gloria ben poco spirituali. Ecco quindi che $i$ temi assunti nelle Prediche d'Amore rimontano alla tradizione elegiaca (Properzio, El. II I, 57-58: Omnes humanos sanat medicina doloris; / solus Amor morbi non sanat artificem; Ovidio, Ars 3, 4I: Quid vos perdiderit dicam; nescistis amare), o al Virgilio bucolico (Omnia vincit Amor, et nos cedamus Amori, Buc. X 69), o al testo evangelico, se può facilmente essere letto in chiave erotica (come Math. 22, 39: Diliges proximum tuum sicut te ipsum). ${ }^{16}$

3.2. Favoriscono la percezione delle Prediche d'Amore come un genere unitario, al di là delle diverse realizzazioni, in prosa o in versi, alcuni indizi testuali e paratestuali, a partire dalla consapevolezza di genere dimostrata da autori e stampatori. La coscienza di adottare la forma della predica trova una prima conferma nei titoli dei testi che ci sono pervenuti. Siamo sempre di fronte, infatti, a un'esplicita titolatura (vedi anche oltre): Predica de amore nel Compendio de cose nove per la predica di Rosiglia (lo stesso Compendio ne porta l'annuncio nel titolo del frontespizio, cosi come le stampe delle opere di Rosiglia) $;{ }^{17}$ una Predica d'amore bellissima è quella composta per el digno poeta Baldoino Cortonense (e più avanti nel testo l'opera è detta Sermo devotissimus); due Prediche d'amore carnascialesche sono quelle di Pier Luigi di Francesco Magdoli, come è ribadito nel corpo della stampa (Predica Prima e Predica seconda); Predicha d'Amore è detta la frottola del Marciano It. IX 310 (6650), Predica de Amore è quella «composta per el Verde Lauro», e «novamente stampata» è la Predica d'Amore adespota conservata a Wolfenbüttel.

Inoltre, anche nel corpo del testo, le Prediche sono corredate da didascalie che ne descrivono la struttura, in termini non sempre identici, ma molto simili tra loro: Oratio $o$ Invocatio, Salutatio, Thema, Thematis repetitio $o$ replicatio (nei casi, evidentemente, in cui il tema è ripetuto), Proemio o Exordio, indicazioni di parte (prima, seconda, terza parte).

Tutto il sistema del "paratesto" concorre, insomma, a definire in termini omogenei l'ispirazione omiletica e la forma propria del sermo.

3.3. Al di là delle definizioni esterne, $i$ testi stessi si dimostrano coscienti della struttura omiletica, e in particolare della struttura del sermo modernus, vuoi con dichiarazioni esplicite sull'occasione predicatoria, vuoi con accenni più sottili, che danno quasi per assodata la forma parodica. Spesso sono gli stessi predicatori d'Amore, che, con dichiarazioni esplicite o con accenni alla tecnica omiletica, si dimostrano consapevoli di utilizzare un genere che rimonta a un 
ipotesto parodiato. Tali accenni non sono da leggere soltanto in chiave metaletteraria, come momenti di riflessione sulla forma che si adotta, ma piuttosto come spie di una dichiarata volontà di adeguamento a un genere.

Ad esempio, per quanto riguarda thema $e$ divisio, $i$ predicatori parodici inseriscono formule destinate a richiamare l'attenzione sulla struttura della predica e non mancano di far notare che proprio a un'operazione di sviluppo tematico si sta procedendo, non diversamente dall'abitudine, diffusa tra $i$ predicatori sacri, alla partium declaratio, alla descrizione della predica che si sta per pronunciare, a fini per lo più mnemonici. Sono inserti che fanno riferimento, più o meno esplicitamente, a una pratica precisa e nota agli ascoltatori, e si pongono quindi come indizi di parodia: il destinatario che sente rievocare formule e forme a lui familiari, interpreta il loro uso nel contesto straniato proprio come volontà di parodia. Un esempio in Rosiglia (Proemio, vv. 3I-48), ${ }^{18}$ che illustra in apertura di Predica le parti in cui strutturerà il suo discorso:

$$
\begin{aligned}
& \text { unde, per far migliore et più saldo processo, } \\
& \text { divideremo expresso in quatro parte, } \\
& \text { le qual con nobil arte provaremo. } \\
& \text { Et questo esser diremo: si est, quid est, quia est } \\
& \text { necnon propter quid est, et in tal forma } \\
& \text { udirete la norma in dolce rima. } \\
& \text { Vedreti ne la prima - et qui l'ingegno inalza - } \\
& \text { se Amor è cosa falsa, o cosa vera: } \\
& \text { dilectevol matera ad tutti voi. } \\
& \begin{array}{c}
\text { Prosoponendo poi esser Amor per certo, } \\
\text { ne la seconda aperto et chiar vi fia } \\
\text { Amor che cosa sia et sua facella. }
\end{array} \\
& \text { Ne l'altra particella vi sarà dechiarato } \\
& \text { Amor donde sia nato et in che modo, } \\
& \text { dissolvendo ogni nodo de ignoranza. }
\end{aligned}
$$

Inoltre, tra gli elementi caratteristici della predica sacra, uno dei tratti più tipici, più immediati da percepire e quindi più facili da assumere come oggetto di parodia, è l'utilizzo che fa il predicatore di prove volte a definire e confermare gli assunti della sua esposizione. Le artes praedicandi avevano stabilito una precisa casistica dei mezzi più idonei allo sviluppo probatorio della 
predica, che non poteva lasciare nulla di vago, di non dimostrato e di poco suadente. Gli strumenti che più sono impiegati in questa operazione di dilatazione del discorso sono sostanzialmente tre: la citazione di auctores, l'innesto di episodi narrativi (exempla), la strutturazione, consequenziale e logicamente ordinata, di argomentazioni, con prove di ragione (rationes, che però nelle Prediche d'Amore sono decisamente minoritarie).

In Rosiglia la scelta dell'accumulo di exempla come occasione didattica è coscientemente adattata e esplicitamente motivata dal predicatore, che dice di preferirla ad altre tecniche di prova (come potrebbero essere le rationes, o le auctoritates), perché più varia e interessante, e meno noiosa (Prima pars, $I-9)$ :

$$
\begin{aligned}
& \text { Potria farvi al presente auctorità molte, } \\
& \begin{array}{l}
\text { in prose et sciolte } \quad \text { oratione: } \\
\text { tropo longo sermone } \quad \text { al mio proposto. }
\end{array}
\end{aligned}
$$

Ma perché io son disposto non esser tedioso al vostro glorïoso et degno aspetto, ho fatto un mio concepto, et fors'è sano

farvi tocar con mano che Amor in noi si trove, solamente con prove et con exempli tratti d'antiqui templi et di moderni.

Spesso, gli exempla delle Prediche d'Amore sono brevi, o brevissimi, limitati talvolta alla mera citazione del nome o al tratteggio rapido delle linee fondamentali della vicenda ricordata: l'effetto probatorio è ottenuto grazie all'accumulo a cascata di casi ben noti, inerenti tutti a un comune denominatore (ad esempio per gli amori conclusi tragicamente si citano Didone, Fillide, Fedra). La forza di un tale tipo di exemplum risiede nella capacità evocativa del nome celebre, specializzato: pressoché tutti questi riferimenti sono tratti dal mondo classico, mitologico, e storico o leggendario. Questo tipo di exemplum "onomastico", per il quale il nome basta da solo a rievocare l'episodio intero, conta tra le sue fonti privilegiate l'Ovidio delle Metamorfosi $e$ delle Heroides, ma è evidente la mediazione dei Trionfi petrarcheschi. Nella forma di predica costruita sulla base di distinctio e di dilatatio, si rendeva poi necessario per il predicatore costruire una fitta rete di rimandi scritturali. Tra i più comuni procedimenti di dilatazione consigliati dai trattatisti medievali, un ruolo importante è ricoperto dalla catena di autorità, "concordate verbaliter, cioè per il ricorrere di una stessa parola o di una medesima radice verbale, o realiter, cioè per l'analogia dei concetti, o per entrambi $i$ motivis. ${ }^{19}$ Nelle Prediche d'Amore si trovano due principali forme di utilizzo della citazione di auctoritates: le citazioni formano quasi 
per intero il corpo della predica, o sono introdotte solo nei punti opportuni come vero e proprio strumento di prova. Vario è il repertorio: nel caso di citazione effettiva domina il patrimonio classico latino, mentre, dove la citazione si riduce alla menzione del nome dell'autore, si elencano in quantità anche Padri della Chiesa e filosofi classici e scolastici (senza allegare il testo), o autori inventati ad hoc.

Di fronte a questa insistenza, anche l'incidenza di auctoritates in alcune Prediche d'Amore è a tutti gli effetti parodica con funzione satirica, con modalità diverse (al limite contrarie) tra gli esemplari in versi e quelli in prosa. Nelle prediche in versi, quando la citazione si limita a un accumulo di nomi di auctores, senza riportarne $i$ testi, la parodia si attua per riduzione, abolendo, di fatto, la stessa auctoritas e mettendo così in luce la vacuità della citazione; nei testi in prosa, dove la citazione di auctoritates costituisce lo scheletro stesso della predica, l'effetto parodico si registra innanzitutto come risposta a un preciso modus operandi di alcuni predicatori, ma in secondo luogo, per quell'accumulo abnorme e dilagante di verità autorevoli, ogni citazione finisce per godere di pari dignità e per perdere l'incisività che le è propria. Le citazioni di auctores sono inoltre le sedi privilegiate per introdurre il latino, di norma corretto, ma spesso franto e allusivo, nel corpo del testo volgare; oltre che nelle citazioni, il latino compare anche in formule discorsive $e$ di snodo del ragionamento, di stampo scolastico (et primum, unde, seu potius, ut inquit...), ma non si arriva mai al maccheronico, né agli estremi di ibridazione dei sermoni mescidati. Come tutto il sistema della predica, anche il latino partecipa degli intenti parodici: il predicatore imita il tono serio delle allegazioni in grammatica, utilizza il latino per nobilitare scherzosamente il discorso, per ironizzare, di fatto, sul suo stesso tono dottorale. A questi aspetti tipici di un sermone, si possono poi aggiungere alcuni topoi: procedono nella direzione della definizione della finzione omiletica tutte le esortazioni che il predicatore indirizza al suo pubblico, come le richieste di silenzio (Seconda parte, 84: «silentio benigno - vi domando»), le promesse di brevità, le domande retoriche, le allocuzioni (Seconda parte, 73: «Adunque, figliol mio, - sa' tu che cosa è Amore?»; Quarta parte, 3: «e perbò [io] v'invito - a star attenti»). Sono inserti che, collegandosi esplicitamente alla situazione della predica, ricordano al destinatario che proprio a un sermone sta assistendo: contribuiscono cosi a fissare i caratteri della fiñione e promuovono l'efficacia parodica del testo. Anche il finale delle Prediche d'Amore, che sono pur sempre costruite per spingere a dedicarsi ad attività erotiche presentate come perfetto compimento della volontà di Dio, ricalca fedelmente il finale delle prediche serie, con la benedizione e la promessa di salvezza eterna. Se Rosiglia scrive (Quarta parte, vv. 86-90): 


$$
\begin{gathered}
\text { Così facendo, in pace et unïone } \\
\text { et in consolazione, in tutte gratie, } \\
\text { le vostre menti satie sarano in questa vita } \\
\text { de [quel]la, ch'è infinita et summa, gloria, } \\
\begin{array}{l}
\text { a la qual con victoria vos perducat Dominus }
\end{array}
\end{gathered}
$$

non può non richiamare alla memoria simili finali, ad esempio, di san Bernardino ( $E$ E però volendoti tu salvare, seguitando quello che t'è stato dimostrato, arai di qua la grazia, e di là arai la gloria; ad quam ille vos et me perducat per infinita secula seculorum. Amen», XXXII 86). ${ }^{20}$

3.4 Il tratto formale che nel corpus delle Prediche d'Amore per primo si impone all'attenzione è l'alternanza di testi in prosa e di testi in versi. L'oscillazione tra prosa e versi non pregiudica la considerazione delle Prediche d'Amore come un insieme unitario e organico; siamo cioè di fronte a un unico genere, che, anche se viene modulato in forme diverse, dimostra tratti coerenti, precisi e che travalicano i confini prosa/verso.

Le prime attestazioni di Predica d'Amore sono in prosa: cosi le Prediche dei ms. quattrocenteschi Magliabechiano VII I0зо e Marciano It. IX III; $i$ testi in versi sono in testimoni più tardi (il Compendio del I507, molte stampe popolari). Ancora nel Cinquecento, tuttavia, si scrivevano Prediche in prosa (come nei casi di Filippo Baldacchino e di Francesco de' Canti).

Allinterno di ciascun sottoinsieme (Prediche in prosa, Prediche in versi), le Prediche presentano alcuni aspetti singolari, più tipici dell'una che non dell'altra manifestazione del genere. Ad esempio, le Prediche in versi condividono, al loro interno, l'appartenenza a un comune clima frottolesco, e sono più esplicite nell'esortazione al sesso. Le Prediche in prosa si caratterizzano, dal canto loro, per un impiego maggiore di dimostrazioni razionali, più minute e strutturate che non negli esempi in versi, e per una spiccata propensione all'utilizzo di auctoritates reali e non fittizie, ben articolate e coerenti, fino a costruire sul loro accumulo lo scheletro stesso della Predica. Un tale impiego di auctoritates è, naturalmente, più facile da gestire in un testo in prosa che in un testo in versi: non a caso, nelle prediche in versi, le auctoritates sono citate brevemente, o subiscono un processo di versificazione che rielabora il dettato originario. Comune alle realizzazioni in prosa e a quelle in versi, e garante di una comune linea di appartenenza, è però la strutturazione della predica in partes, articolate su un thema di base. Il riferimento parodico comune a uno stesso genere porta alla condivisione di tratti costanti, che vengono declinati con inevitabili aggiustamenti tanto nelle prediche in prosa quanto nelle prediche in versi; le une e le altre, infine, fanno riferimento a occasioni carnevalesche e festive. 
La Predica d'Amore aveva come suo referente principale un genere in prosa (anche se non sono ignoti casi di sermoni in versi). Se la prosa poteva essere quindi il mezzo più immediato di espressione, la versificazione poteva trovare nella tradizione carnevalesca, di piazza, o in senso ampio satirico-frottolesca, un sicuro incentivo o una sollecitazione alla "traduzione" formale. L'assunzione della forma in prosa piuttosto che in versi, non sembra, per altro, implicare particolari prese di coscienza dell'autore, che probabilmente disponeva dell'uno e dell' altro mezzo, senza determinati obblighi di allineamento a norme esclusive.

Tutte le Prediche d'Amore in versi, pur nelle differenze di metro che corrono tra l'una e l'altra, fanno comune riferimento a un modello di frottola regolarizzata, come si era sviluppata nel corso del Quattrocento e risentono dell'influsso della zingaresca e dell'endecasillabo frottolato. ${ }^{21}$ I metri sono quindi settenari a rima baciata, endecasillabi frottolati, zingaresche - non sempre regolari, come nel caso delle Prediche di Magdoli: ma l'andamento disordinato del metro può essere una caratteristica propria del testo, indirio di una composizione distratta o di una rielaborazione poco attenta. Non è escluso, del resto, che in una possibile recitazione del testo i problemi di natura metrica venissero sfumati nell'esecuzione.

La vicinanza della Predica alla frottola si colora di un'ulteriore suggestione. La frottola stessa, infatti, si dimostra fin dalle origini (e fino alla regolarizzazione quattrocentesca) carica di una ambiguità di fondo: come è noto «la maggior parte delle frottole furono [...] scritte e trascritte a mo' di prosa». ${ }^{22}$ Anche Berisso, indica "uno statuto per cosi dire 'ibrido' della frottola, sequenza ininterrotta avvicinabile alla prosa e probabilmente deputata ad una lettura ad alta voces. ${ }^{23}$ Più recentemente, anche Giunta ritiene che la frottola possa essere considerata all'incrocio «tra la poesia liberata dalle maglie della prosodia e la prosa rimata». ${ }^{24} \mathrm{Ci}$ si può chiedere, se questa natura quasi prosastica della frottola non abbia in qualche modo facilitato l'assimilazione prosa/versi per un genere come la Predica d'Amore, che, derivato per via parodica da una forma in prosa, si concretizzava anche in realizzazioni in versi, in toni burleschi e faceti.

4. Tutto l'impianto delle prediche è rivolto, si è visto, ad esaltare il potere di amore, a dimostrare la necessità di porsi al suo seguito, ad esaminare accuratamente il tema, a spingere a cedere al piacere carnale. Questo intento parenetico, che ricalca la reale prassi omiletica, si spinge fino alla mimesi delle concrete applicazioni del testo, destinato verosimilmente a un'esecuzione orale. La Predica d'Amore si finge recitata o letta dal predicatore di fronte al suo pubblico. I segnali dell'intenzione orale sono piuttosto frequenti: possono anche essere un semplice adeguarsi alle conven- 
zioni e alle circostanze della predicazione seria, ovviamente agita di fronte a un uditorio, ma è molto verosimile pensare che la finzione parodica si spingesse fino alla rappresentazione; in questo senso, lo fanno credere $i$ numerosi riferimenti alla dimensione della parola pronunciata e dell' ascolto (Proemio, 5I: «quieta audientia - mi prestate»; Prima parte, 103-104: «Cosi da parte mio - parlar porremo / et l'altro pigliaremo - senza far posa»). E del resto, in una circostanza festiva, è facile immaginare che l'esecuzione fosse stata piuttosto orale, comunitaria, piuttosto che essere affidata alla lettura individuale.

L'esecuzione orale, festiva, attestata del resto anche per le realizzazioni colte di letteratura carnevalesca come le Stanze di Bembo e le Farse di Sannazaro recitate durante il carnevale, è coerente anche al tipo di pubblico e ai canali di diffusione delle prediche, che sembrano polarizzati su due versanti, l'uno più popolaresco (la fiera, la piazza), ma che concerne sempre un destinatario in grado di decifrare il gioco parodico; l'altro più colto e letterario (la corte, il mondo urbano). Si tratta di due versanti in rapporto tra loro, come sempre più si riesce a dimostrare a proposito di manifestazioni letterarie tributarie in qualche modo dell'universo popolare: due linee di tradizioni in stretto dialogo tra loro. ${ }^{25}$ Sembra dunque verosimile prospettare anche per le Prediche d'Amore una situazione simile a quella, di alcuni decenni posteriore, studiata per Giulio Cesare Croce, per il quale è accertata la lettura da fiera, e il doppio registro di pubblico, popolare e colto, sia al livello della comunicazione orale, sia al livello della trasmissione scritta. ${ }^{26}$

5. Quanto alla loro diffusione, le Prediche d'Amore sono trasmesse da manoscritti e da testi a stampa. Spesso si tratta di testimoni unici, ma almeno nel caso della Predica attribuita a Marco Rosiglia la tradizione è mista. Il codice della Biblioteca Estense Universitaria di Modena, Campori I 87 (su cui vedi oltre), è una silloge di testi cortigiani (ma con il primo Ariosto e il primo Bembo). Come il suo parallelo a stampa, il Compendio di cose nuove, con cui condivide, senza rapporti di derivazione diretta, alcuni testi tra cui la Predica d'Amore, è rappresentativo del clima poetico precedente alla codificazione più rigidamente bembesca, e indica il lato più colto, aristocratico del genere predica, divertimento non solo da fiera, ma in voga anche presso circoli colti. I manoscritti della Biblioteca Marciana sono testimoni del clima letterario quattrocentesco come l'It. IX III $(6358)$ che contiene anche un canzoniere quattrocentesco, o della curiosità eclettica di metà cinquecento, come lo zibaldone It. IX 310 (6650), che raccoglie alcuni testi della tradizione comica, con capitoli burleschi e canti carnascialeschi. Il ms. Magliabechiano VII I0zo, veneto, contiene testi di letteratura popolaresca, tra cui tre mariazi, editi recentemente da Milani. ${ }^{27}$ 
Sull'altro versante, $i$ testi a stampa: testi a circolazione ampia ma orientata, come il Compendio di cose nuove, vero e proprio "compendio" formale e tematico del gusto e della poesia cortigiana al passaggio tra Quattro e Cinquecento, o come la silloge delle opere di Rosiglia; e testi a circolazione più indiscriminata, che si potevano smerciare nelle fiere e nelle piazze come i fogli volanti e le stampe popolari, arricchite in alcuni casi di qualche rispetto o di un capitolo amoroso. Il mercato di riferimento è quello dei librai, dei cartolai e dei cerretani, produttori di testi velocemente confezionati e velocemente consumati che rispondono a esigenze di vendita e a richieste di materiale facilmente godibile. Di qualità spesso scadente per la loro natura di prodotto anche materialmente fragile, sono giunti a noi per vie avventurose: il che spiega come molte delle Prediche d'Amore non ci siano note che in testimone unico.

6. La storia critica delle Prediche d'Amore inizia già con Dolce che nel Dialogo dei colori, cita la Predica contenuta nel Compendio quando cerca una definizione della frottola.

Cornelio. Fu il Calmeta con pace sua goffo: e se bene s'interteniva alla corte di Urbino a tempi, che vi fioriva il Bembo, il Castiglione, \& altri simili huomini, non è che per tale egli non fosse havuto. Chi mandasse adunque a donare le cose sue, tratterrebbe colui, a cui le mandasse senza fallo da goffo.

Mario. Fece pure egli quella frottola, che incomincia:

Omnia vincit Amor; \& nos cedamus amori.

A Pastore Pastori

In Buccolicis scriptum,

Pulchrum Poetae dictum Mantuani

Connelio. Non fu questa frottola, ma predica. Ma non è maraviglia, che egli la fece per giuoco. È da maravigliarsi del Petrarca, che havendo così purgate orecchie scrivesse questi versi:

Di rider non ho voglia,

Per una grave doglia,

Che m'è nata nel fianco

Di sotto al lato manco.

Benchè non tanto è da maravigliarsi, che egli facesse questi bassi versi, quanto da stupire di quegli altri, ne' quali questa sua frottola mutò:

Mai non vo più cantare, come io soleva

Ch'altri non m'intendeva, ond'hebbi scorno:

E puossi in bel soggiorno esser molesto. ${ }^{28}$

L'attribuz̨ione a Calmeta, nella relativa scarsità di testi di sua mano, è certo suggestiva, e l'aria che si respira in alcune prediche non è molto diversa da quella del suo compendio dell'Ars amandi. Ma l'attribuzione a Rosiglia 
sembra preferibile, posta la presenza della Predica nella raccolta delle rime di quest'ultimo (e ammessa l'attrazione che il nome più celebre del Calmeta può aver esercitato su Dolce).

Le riserve sul valore artistico della predica si ritrovano in alcuni gindizi e informazioni sulle Prediche d'Amore nelle opere erudite del Settecento, che spesso ripetono errori e indicazioni confuse. Cosi Quadrio, Della storia e della ragione d'ogni poesia, II, I74I, Libro I, Dist. I, Capo VIII, p. 2I7, a proposito di Calmeta cita un'edizione del Compendio:

Vincenzo Calmeta, da Castelnuovo, fu buon letterato de' tempi suoi. Scrisse un Trattato della Volgar Poesia, il Pellegrinaggio d'Amore, e diverse Rime, nelle quali però fu poco felice, se non vogliamo anzi col Dolci chiamarle sciaurate. Furono queste impresse in Venezia per Zorzi de' Rusconi nel I 507, in 8. col titolo: Opera nuova di Vincenzo Calmeta, L. Carbone, Orfeo Mantovano, ed altri Autori: e di poi ristampate pur quivi per Simone de Luere nel i 5 I 4. in 8., con quest'altro Titolo: Compendio di cose nobile, et delectevole de Vincenzo Calmeta, et de altri Auctori, cioè Sonetti, Capitoli, Epistole, Egloge pastorale, Strambotti, \& Barzellette con una Predica d'Amore. [...]

attribuendo la Predica, sulla scorta di Dolce, allo stesso Calmeta (p. 218):

La Predica d'Amore, che va senza nome di Autore nella citata edizione, è dal Dolce nel Dialogo de' Colori attribuita allo stesso Calmeta, che in un'altra Raccolta impressa per lo Zoppino nel i 2 I, viene soprannominato De Collis.

Nel 1747, interviene Cinelli (Biblioteca volante, tomo IV, p. I74), a proposito di una Predica stampata autonomamente, e la attribuisce a Rosiglia (Cinelli sembra non conoscere la Predica contenuta nel Compendio):

Rosiglia (Marco), Predica d'Amore molto piacevole e bella nuovamente ristampata. In Firenze nel 1556 . del Mese di Novembre in 8. In questa edizione di Firenze non vi si vede punto il nome dell'Autore, ma è certissima cosa ch'ella è componimento di Maestro Marco Rosiglia da Fuligno, trovandosi stampata più volte, col suo nome nel libro dell'altre sue Poesiae; io però non poco mi maraviglio, ch'in Firenze si permettesse, che si stampasse un Componimento tanto licenzioso per non dire altrimenti.

Nel 1752, Quadrio ritorna sulla sua nota e a proposito delle informazioni sulla predica del Compendio, che seguendo Dolce aveva già attribuito a Calmeta, (p. 66) aggiunge:

Ma essa è opera di Filippo Baldachino, come in appresso vedremo.

Quadrio fa confusione, ingannato, si immagina, dal solo titolo - visto che le informazioni che darà a proposito della Predica di Baldacchini sono 
sostanz̧ialmente aderenti alla Predica, per l'appunto, di Baldacchini, ma certo non alla Predica del Compendio. Nelle righe dedicate a Baldacchini, dopo una nota sul suo Protocinio, si legge una precisa descrizione di un'operetta in prosa (Nox illuminata). Quadrio, Indice universale I752, p. 67:

La Notte Illuminata, e Correzione della Predica fatta sotto il titolo d'A more dello Stesso, In Firenze per Bartolommeo da Brescia [Bartolomeo Zanetti] i 5 I9, in 8. E prima vi ha la Notte illuminata, il cui titolo è proposto nel Frontispizio in Lingua Latina con queste parole: Philippi Baldachini Coritani Nox illuminata. Prima però di tal Opera si legge una Lettera Latina a Silvio Passerino da Cortona Cardinale di S. Chiesa, e Datario di Leon X. data a' i3. di Agosto del 1715. dalla Vecchia Accademia Gregoriana (ex Veteri Gregoriana Accademia) cioè di Gregorio Leti. Alla Lettera succede un' altro [sic] nuovo Titolo, che è tale: Sermo piissimus babendus in Liberi Patris festivitatibus, toto Orbe celebrari consuetis, ad amentes, \& rudes, ut amantes \& sapientes fiant. Questa è la Predica d'Amore piena di molte sciocchezze, e di poche onestà. Alla Predica succede la Correzione col titolo Correctio Praedicationis sub titulo Amoris factae, dove si ritratta tutto ciò che di licenzioso e di scostumato si è detto nella Predica d'Amore.

Le notizie di Quadrio passano integralmente a Mazzuchelli, che ne riporta con fedele aderenza anche $i$ giudizi di valore. Mazzuchelli, Gli scrittori d'Italia, 1758, Vol. II, Parte I, p. 93-94:

BaldacChini (Filippo) Coritano (cioè Cortonese) Poeta Volgare, [...] ha composto le seguenti Opere, le quali oltre all'essere insulse e piene di sciocchezze, sono anche poco oneste.

I. Nox illuminata (ovvero Predica d'Amore con la Retrattazione della medesima. Florentiae datum VII. Kal. Novemb. I 19 . opera \& cura vigili Bartholomei Brixiensis, in 8. A quest'Operetta, ch'è mescolata di Latino, e di Volgare, sta premessa una Lettera Latina dell'Autore al detto Card. Silvio Passerini segnata ex veteri Academia Gregoriana 23. Augusti I 5 I 5 . Alla Lettera succede un'Operetta intitolata: Sermo piissimus habendus in Liberi Patris Festivitatibus, toto orbe celebrari consuetis ad amentes \& rudes, ut amantes \&s sapientes fiant. Questa è la Predica d'Amore, la quale non merita d'esser letta, essendo e insulsa e licenziosa. Alla Predica succede la Correzione della medesima col titolo: Correctio Praedicationis sub titulo Amoris facta, dove l'Autore ritratta tutto ciò che di poco onesto e scostumato ha detto nella Predica d'Amore.

Ancora tra gli eruditi, il Melzi, Dizionario degli Anonimi, vol. II, p. 270 (a proposito dell'Opera nuova), ricorda le diverse attribuzioni della Predica del Compendio a Calmeta e a Rosiglia, ma per identificarla poi erroneamente con la Nox illuminata di Baldacchini: Dizionario di opere 
anonime e pseudonime di scrittori italiani o come che sia aventi relazione all'Italia, Milano, Pirola, $1848-1859$ :

Opera nuova di Vincenzo Calmeta, L. Carbone, Orfeo Mantovano, ed altri autori. Venezia, per Zorzi de' Rusconi, I 507, in 8., ed ivi ristampata per Simone de Luere, I I I4, in 8. con quest'altro titolo - Compendio di cose nobile et delectevole de Vincenzo Calmeta, et de altri auctori, cioè sonetti, capitoli, epistole, egloghe pastorale, strambotti et barzellette, con una Predica d'Amore.

In tal raccolta non si sa chi sia quel L. Carbone, e di chi sia la Predica d'amore. [...] La Predica d'amore fu anche ristampata a parte senza nome d'autore con questo titolo - Predica d'amore molto piacevole e bella, nuovamente ristampata in Firenze nel I 556, del mese di novembre, in-8. Gli annotatori della Storia della volgar poesia del Crescimbeni, appoggiati dall'autorità di Lodovico Dolce, l'attribuiscono a Vincenzio Calmeta. Il Cinelli la crede di Marco Rasilia, perché sotto il nome di lui leggesi in una raccolta di poesie di più autori, impressa dal Zoppino, senz'anno, e della quale ei fece una ristampa nel i s i . Noi diremo che l'autore di detta Predica è Filippo Baldacchins, come apparisce dal catalogo Capponi e del Mazzuchelli (Scritt. d'Italia) che riportano la seguente edizione - BALDACCHIn (Filippo), Coritano (cioè Cortonese), Nox illuminata, ovvero Predica d'amore, con la ritrattazione della medesima. Florentiae, datum VII kal. novembr. I 5 19, opera et cura Bartholomaei Brixiensis, in 8. Precede una lettera latina dell'autore al Card. Silvio Passerini in data del 25. agosto i 5 I 5 . Questi due insulsi opuscoli sono un miscuglio in gran parte di volgare e di latino. Alla sciocchezza, il primo di essi, aggiunge anche la disonestà.

Confondono anche alcuni brevi interventi sul Giornale degli eruditi e dei curiosi, II (p. 496):

Più di proposito parla di quest'operetta il Melzi nel Dižionario degli Anonimi II. 270 , il quale ci fa sapere che fu anche ristampata senza nome d'autore col titolo di Predica d'amore molto piacevole e bella, nuovamente ristampata in Firenze nel i 556, del mese di novembre. Ora io aggiungo che n'esiste anche una terza edizione collo stesso titolo di Predica d'amore nuovamente stampata, senza data, di 4 carte in 4 . Comincia

Omnes humanos sanat / medicina doloris

Pregoti abbi pietade De tuoi poveri amanti

Poi Proemio - Prima pars - Seconda pars. Ogni parte finisce: Sputate che ancor sputo. - Tertia pars, finisce Che amor vi benedica. Questo cenno, che trovo fra i miei appunti, basta per assicurarsi trattarsi di una vera sciocchezza.

S. B.

Si tratta qui di una predica anonima conservata a Wolfenbüttel, che non ̀̀ evidentemente una terza edizione del testo, ma una terza, diversa predica. 
Sempre nel Giornale degli eruditi e dei curiosi, pp. 6I8-62I, Tessier fa un po' d'ordine e distingue, giustamente, tra la predica di Rosiglia e la predica di Baldacchini. Tessier cita l'Opera nova del precarissimo Marco Rosiglia (Rusconi, Io gennaio III6), ne riporta alcuni versi ("Potria farvi al presente - autorità molte»; «Alcuno degno autore - ch'amore ba definito»), $e$ mette infine in chiaro che «il componimento del Rosiglia è cosa affatto diversa di quello del Baldacchini» (p. 620).

Nello stesso Giornale degli eruditi e dei curiosi del I883, p. 436 Alessandro D'Ancona interviene con una breve nota bibliografica («Nox illuminata. Qualche cenno su questo libro è da trovarsi nel Catalogo della Libreria Capponi 1747, pag. 42, Pisa»). D'Ancona aveva già del resto provveduto a offrire la descrizione di una predica di Wolfenbüttel, che si legge nell'opuscolo Due Farse del secolo XVI riprodotte sulle antiche stampe. Con la descrizione ragionata del volume Miscellaneo della Biblioteca di Wolfenbüttel contenente Poemetti popolari italiani compilata dal Dott. G. Milchsack con aggiunte di A. D'Ancona, Bologna I882, p. 2I4-IS.

La Predica d'Amore è stata infine recentemente riportata all'attenzione, quando nel 1981 Maria Teresa Casella e Giovanni Pozzi pubblicarono una Predica in prosa che l'unico testimone (Magliabechiano VII 1030) attribuisce a Francesco Colonna, autore dell'Hypnerotomachia Poliphili. Casella e Pozzi collocano attentamente la Predica nel suo sfondo storico, ma il loro interesse è rivolto al problema attributivo: come dichiarano proprio in apertura di intervento, $i$ curatori pubblicano il testo «non per qualche suo merito di contenuto o di lingua, ma per il nome che vi figura in testa sul solo codice che lo trasmette: nientemeno che quello di Francesco Colonna». ${ }^{29}$

\section{La predica attribuita a Marco Rosiglia}

La maggior parte delle Prediche sono note da pochi testimoni: fa eccezione la Predica di Marco Rosiglia, che si legge, come già si accennava, in sedi diverse: nel Compendio del I 507 e sue ristampe, nelle raccolta delle Opere di Marco Rosiglia, in stampe popolari autonome, in un testimone manoscritto non dipendente dalle stampe note. Gran parte del successo editoriale della Predica è dovuto alla sua collocazione nel Compendio di cose nuove, libretto di vasta diffusione (almeno otto ristampe nel giro di quindici anni), e poi nell'edizione delle Opere di Rosiglia, ma non è da sottovalutare la sua presenza in almeno tre diverse edizioni di stampe popolari. Il relativo successo conosciuto da un autore minore come Rosiglia può essere spiegato anche in ragione di opportunità editoriali: nei primi decenni del Cinquecento si stava affermando la fiera del libro di Foligno, città natale di Rosiglia, e non è escluso che in occasione della fiera si moltiplicassero le possibilità del commercio locale di un 
nome localmente attraente (tra l'altro, alla fiera di Foligno è accertata la presenza dello Zoppino, stampatore non solo del primo Compendio ma anche di due diverse edizioni di rime di Rosiglia)..$^{30}$

La predica è in versi di ascendenza frottolesca, in strofe simili alle strofe della zingaresca e di alcune frottole, con il raddoppiamento dell'endecasillabo di chiusura della strofa (dato dalla fusione di due emistichi, settenario + quinario/quadrisillabo):

$\begin{array}{lll}\text { zingaresca } & 7+7+7+4 / 5 & =\mathrm{abbc}_{4 / 5} \\ & 7+7+(7) \mathrm{II} & =\mathrm{ab}(\mathrm{b}) \mathrm{C} \\ \text { predica } & 7+7+(7) \mathrm{II}+(7) \mathrm{II} & =\mathrm{ab}(\mathrm{b}) \mathrm{C}(\mathrm{c}) \mathrm{D}^{3 \mathrm{I}}\end{array}$

La predica è costruita sul tema Omnia vincit Amor, et nos cedamus Amori (Virgilio, Buc. $x$ 69); articolata in quattro parti, precedute dall'esposizione del Thema, dall'Oratio ad Venerem (ottava: "Venere, radiante stella in cielo»), dalla Thematis repetitio, con la riproposizione del tema, da un Proemio con l'esposizione del tema e della struttura della predica.

L'attribuzione della Predica non è del tutto scontata (Dolce, come si accennava, la attribuisce a Calmeta; nel Compendio e nelle stampe popolari è adespota), ma è rafforzata dalla presenza nelle raccolte personali, non miscellanee, di Rosiglia, per quanto postume. ${ }^{32}$ Posta la paternità, rimangono però alcune zone d'ombra sulla figura di Marco Rosiglia, medico di Foligno (il nome a volte compare come Rasiglia). Ludovico Iacobilli, nella sua Bibliotheca Umbriae scrive:

Marcus Rosilius Fulginas, Vagnoni Bernardoni filius e Regione Crucis, Philosophus, Medicus, Poeta, ac Canonista celebris; qui obiit Fulginiae die i 5 . Novemb. an. I 508 . Edidit ordinationes divini Officii totius anni. Romae an. I 503 in 8. plura super Medicinam; novella Poemata utroque carmine, praesertim Sopra la vita di s. Maria Madalena, di s. Marta, e di s. Lazzaro in ottava rima, pluries impressa in variis locis. ${ }^{33}$

Non dice molto Michele Faloci Pulignani in un intervento sul "Giornale storico della letteratura italiana», dedicato alla letteratura a Perugia nel XV secolo. ${ }^{34}$

Gli interessi letterari di Rosiglia dovettero essere coltivati alla corte di Urbino. Nelle sue opere si tramanda un lungo capitolo in terza rima, che si presenta come un'epistola consolatoria inviata a Elisabetta Gonzaga dal marito morto, il duca d'Urbino Guidobaldo da Montefeltro (morto il 3 aprile I508). Il capitolo è accompagnato da una breve epistola di dedica di Rosiglia a Elisabetta, che dimostra una qualche famigliarità con l'ambiente, anche culturale, urbinate. Ne riporto un passo: ${ }^{35}$ 
[...] trovando per la damnosa morte de lo amato consorte, lachrymosa et obscura, officio de fidel servo, me parve, in quel più grato et honesto modo che ad me fosse possibile, consolarve: la qual occasione forsi audacemente presa, la subsequente epistola feci, dono per la excellentia de la materia et humilità de lo stile et ad voi et ad me congruo in parte. Certo so, ad lo altissimo ingegno et ad vostra poetica academia almancho como l'orso piacerà, el quale per sua goffezza solamente diletta. Ma pur che la mia Epistola in qualche modo da tanta machinatione relevi, serà al mio proposito frutuosa: la quale se da vostri ingegniosi et numerosi poeti più fieramente se castigasse, prego con amorevoleza et misericordia, Signora, gli diate adiuto. Vale.

La composizione per la morte del duca Guidobaldo, oltre che indicare un rapporto almeno con la duchessa Elisabetta, presenta legami illustri con due testi, ben altrimenti autorevoli, che lasciano «trasparire, sia pure in modi diversi, la situazione di reale difficoltà in cui si trova Urbino già sotto Guidobaldo»:36 l'epistola Ad sacratissimum Britanniae regem Henricum, de Guidubaldo Urbini duce di Castiglione e il dialogo De Guido Ubaldo Feretrio deque Elisabetha Gonzagia, Urbini ducibus di Bembo. ${ }^{37}$ Dalla lettera di dedica a Elisabetta, sembra che Rosiglia sia ben consapevole del clima culturale della corte, con la sua «poetica academia» e $i$ suoi «ingegniosi et numerosi poeti»: $e$ in effetti è ben noto che la corte dei Montefeltro era "frequentata occasionalmente da intellettuali di diversa provenienza e cultura». ${ }^{38}$ Anche la richiesta di indulgenza e di soccorso nel caso di un giudizio severo dei poeti di corte, per quanto motivo stereotipato, può essere un ulteriore elemento che depone a favore dell'ipotesi di un reale contatto di Rosiglia con l'ambiente urbinate. ${ }^{39}$

Matteo Largaiolli 
I. Ancora fondamentale per la parodia sacra il quadro storico offerto da Francesco Novati, La parodia sacra nelle letterature moderne, in Studi critici e letterari, Loescher, Torino I889, pp. 177-3 Io. Indicazioni anche in Francesco Novati, Tre lettere giocose di Cecco d'Ascoli, in «Giornale storico della letteratura italiana», I (I 883), pp. 62-74. Più recentemente Guglielmo Gorni e Silvia Longhi, La parodia, in Letteratura italiana, vol. V, Le questioni, Einaudi, Torino I986, pp. 459-87; un utile panorama sulle teorie della parodia in Nella Giannetto, Rassegna sulla parodia in letteratura, in «Lettere italiane», XXIX, 4 (I977), pp. 46I-8I, da integrare con Massimo Bonafin, Contesti della parodia. Semiotica, antropologia, cultura medievale, Utet, Torino $200 \mathrm{I}$.

2. Gorni-Longhi, La parodia, cit., p. 479. Uno snodo decisivo nello sviluppo della parodia volgare nei secoli XII e XIII è stato il nuovo ruolo ricoperto dall'intellettuale nella realtà tardo medievale: un intellettuale «più consapevole delle sue armi critiche, più insofferente delle costrizioni delle norme, dei precetti, dei vincoli, estetici come filosofici, che non derivino da decisioni razionali, ma solo dall'autorità» (Bonafin, Contesti della parodia, cit., pp. I 19-20). L'esempio forse più celebre di parodia del sermone è l'Encomium Moriae di Erasmo da Rotterdam. Sulla fortuna europea della parodia del sermone cfr. Sander L. Gilman, The Parodic Sermon in European Perspective. Aspects of Liturgical Parody from the Middle Ages to the Twentieth Century, Franz Steiner, Wiesbaden 1974, che presta particolare attenzione alla funzione satirica e politica della predica parodica in età di Riforma e Controriforma.

3. In Novati, La parodia sacra, cit., pp. 289-300; Novati la trae dal codice quattrocentesco Vaticano Palatino 719, cc. 5or- 5 Iv: la Missa potatorum ricalca passo passo la liturgia, rileggendo l'azione sacra in chiave bacchica, dal Confiteor al Gloria, alle letture (con la Lectio Actuum potatorum ad Ebrios, che parodizza la lettera ad Hebreos), all' Alleluia con la sequenza Vinum bonum et suave e la Sequentia falsi Evangelii secundum Bacbum, l'Offertorium, il Sanctus, il Pater noster, l'Agnus Dei, e naturalmente la comunione (verosimilmente sotto la specie del vino, prima che del pane) e la benedizione finale. In questa precisa successione liturgica mancano il rito eucaristico vero e proprio con la rievocazione dell'Ultima Cena e la consacrazione (momento forse troppo sacro per farne oggetto di divertimento), il Credo e la predica.

4. Sulle prediche di Carnevale cfr. Piero Camporesi, La maschera di Bertoldo. G. C. Croce e la letteratura carnevalesca, Einaudi, Torino 1976, pp. I69 e sg. Camporesi pubblica in appendice due Prediche di Carnevale (pp. 25 I-82). Carnevale, del resto, nella sua personificazione, è particolarmente produttivo di parodia. Oltre che delle prediche, Carnevale è protagonista di altre forme di parodia: testamenti, confessioni, lettere, orazioni, a dominante mangereccia.

5. La Predica d'Amore non è esclusiva della tradizione italiana. I sermons joyeux francesi, che indagano soprattutto i temi del cibo e del sesso, e spesso sono costruiti come sermoni agiografici, condividono con le Prediche d'Amore il carattere parodico (cfr. Recueil de sermons joyeux, Edition critique avec introduction, notes et glossaire par Jelle Koopmans, Librairie Droz, Genève 1988); ben più stretta la somiglianza con la Predica d'Amore di area spagnola. Il portoghese Gil Vicente scrisse un Sermam pregado em Abrantes, la cui datazione precisa è controversa, ma che è collocabile comunque all'inizio del i 500 , un Sermòn jocoso inserito nell'Auto da Mofina Mendes, un Sermòn de amores nell'Auto da fadas (cfr. Manuel Ambrosio Sànchez Sànchez, Vernacular preacbing in Spanish, Portuguese and Catalan, in The Sermon, a cura di Beverly Mayne Kienzle, Typologie de sources du moyen âge occidental, fasc. $8 \mathrm{I}-83$, Brepols, Turnhout 2000, p. 838, con bibliografia). In prosa è il Sermòn de amores di Diego de San Pedro (morto intorno al I I 13 ), che si legge in Diego de San Pedro, Obras completas, ed. Keith Whinnom, Castalia, Madrid 1985 . Come parte di una Misa de Amor è inteso il sermone in versi del castigliano Francesc Moner (scritto intorno al I485); tracce umanistiche, con citazioni da Aristotele, Ovidio e Petrarca, reca un sermone catalano in prosa di Francesc Alegre (Sànchez Sànchez, Vernacular preaching in Spanish, Portuguese and 
Catalan, cit., pp. 838-39). Il precedente iberico più celebre resta però, pur con pareri discordanti sull'interpretazione che si deve dare al motivo parodico, il Libro de Buen Amor (secondo quarto del tredicesimo secolo), il cui prologo ricalca la struttura del sermo modernus (Janet A. Chapman, Juan Ruiz's «Learned Sermon», in «Libro de buen amom» Studies, ed. G. B. Gybbon-Monypenny, Tamesis Books, London I970, pp. 29-5 I, p. 36. Cfr. anche Gilman, The Parodic Sermon, cit., pp. I 5-16).

6. Monique Rouch, Diffusion orale, fenilles volantes, écrits populaires an XVI siècle: le cas de Giulio Cesare Croce a Bologne, in Autres Italies. La culture intermédiaire en Italie: les auteurs et leur public. Journée d'études du I 3 mars 1993 sous la direction de Monique Rouch, Centre Interuniversitaire de Recherche sur l'Italie: langue, littérature, interactions culturelles, sociétés et mentalités (CIRILLIS), Editions de la Maison de Science de l'Homme d'Aquitaine (M.S.H.A.-CIRILLIS), Talence I994, pp. 31-53, p. 38.

7. Francesco Novati, Tre lettere giocose di Cecco d'Ascoli, in «Giornale storico della letteratura italiana», I (I 883), pp. 62-74, p. 68, che cita Albino Zenatti, Un amore del Muzio e un carnevale a Valperga (Una lettera inedita di Girolamo Mu₹io alla Signora Margherita Tiæia, contessa di Desana), in «Il Fanfulla della Domenica», II (1880), 5, pp. 3-4; la lettera è tratta dal codice Riccardiano 2115 , pp. $589-596$.

8. Le farse di leggono in Iacobo Sannazaro, Opere volgari, a cura di Alfredo Mauro, Laterza, Bari I96r. Gli studi di riferimento per i generi festivi a Napoli sono Mauro Bersani, Alla ricerca dello specifico testuale nelle «Farse» del Sannazaro, in «Lettere italiane», XXXIV (1982), pp. 506-29; Mauro Bersani, Farsa, intermez:o, gliommero. Appunti sul teatro del regno aragonese di Napoli, in «Studi e problemi di critica testuale», 26 (1983), pp. 59-77; Nicola De Blasi, Intrattenimento letterario e generi conviviali (farsa, intermez:o, gliommero) nella Napoli aragonese, in Passare il tempo. La letteratura del gioco e dellintrattenimento dal XII al XVI secolo. Atti del Convegno di Pienza Io-I4 settembre 1991, Salerno Editrice, Roma 1993, tomo I, pp. $129-59$.

9. De Blasi, Intrattenimento letterario e generi conviviali, cit., p. I42. Sannazaro stesso dichiara nella lettera di accompagnamento di una delle sue farse che la composizione era destinata al carnevale: «La farsa del tuo diletto e caro consorte, Ill.ma S.ra mia, fo fatta dui dì da poi de quella del S.or Duca, zoè a' sei marzo, il dì ultimo de carnevale prossimo passato, nello anno del nostro Redentore mcccclxxxxii, e fo il titulo de quella: Il Triunfo de la Fama» (Sannazaro, Opere volgari cit., p. 286). Le farse in questione sono le due composte da Sannazaro in occasione della presa di Granata del $149^{2}$ (La presa di Granata, Il Triunfo de le fama, numeri v e vi dell'ed. Mauro).

ı. Bersani, Alla ricerca dello specifico testuale cit., p. 509.

i i. (Bartolomeo Cavassico), Le rime di Bartolomeo Cavassico notaio bellunese della prima metà del secolo XVI, con introduzione e note di Vittorio Cian e con illustrazioni linguistiche e lessico a cura di Carlo Salvioni, Romagnoli, Bologna I 894 (rist. anastatica Commissione per i testi di lingua, Bologna 1969).

I 2. «De qua non c'è cosa che a quella non sia nuova, eccetto che el Calmeta continuamente compone canzone e diverse opere, e questo carnevale ha fatto una nuova comedia, la quale l'averia mandata a V. Ecc., quando avesse creduto fusse de piacere a quella»: si legge in Vincenzo Calmeta, Prose e lettere edite e inedite, a cura di Cecil Grayson, p. XXXV (da Luzio-Renier, Mantova e Urbino, p. Ioo). Anche Bembo, in una lettera del 20 marzo I 504 a Emilia Pio, nomina l'attività di Calmeta per il carnevale: «Il vostro Centauro ci ha fatto, per sue lettere, partecipe delle feste che eravate per aver questo carnassale per grazie del nostro M. Vincenzo Calmetay: Pietro Bembo, Lettere, edizione critica a cura di Ernesto Travi, vol. I (I 492-I 507), Commissione per i testi di lingua, Bologna 1987 (lettera n. I83, p. 170). 


\section{LA PREDICA D'AMORE}

I 3. Pietro Bembo, Stanze, edizione critica a cura di Alessandro Gnocchi, Società editrice fiorentina, Firenze, 2003 .

I4. Floriana Calitti, Letteratura e svaghi di corte: le Stanze di Pietro Bembo, in Passare il tempo, cit., tomo II, pp. 6I9-3 I, p. 623. L'ispirazione festiva cortigiana è assicurata da una lettera di dedica di Gonzaga a Elisabetta d'Urbino, che precede il testo nel codice Vat. Lat. 8203: «essendo a questo carnasciale passato dal Nobilissimo Cavagliero messer Baldesare Castiglione et da me state composte alchune stanze pastorali le quali, sì come V. Signoria si deve racordare, furono inanti a lei pastoralmente recitate [...] ne è parso racoglierle insieme e mandarle a lei» (Claudio Vela, Il Tirsi di Baldassar Castiglione e Cesare Gonzaga, in La poesia pastorale nel Rinascimento, a cura di Stefano Carrai, Antenore, Padova 1998, pp. 245-92, p. 288).

I s. Un quadro completo sulla predicazione medievale in Carlo Delcorno, Medieval Preaching in Italy (I200-I500), translated by Benjamin Westerwelt, in The Sermon, a cura di Beverly Mayne Kienzle, cit., pp. 449-560, con bibliografia pregressa.

I6. In realtà, non tutte le Prediche riportano un tema citato da una fonte riconoscibile. In alcuni casi, il tema è elaborato ex novo, ma la matrice erotica è sempre riconoscibile.

17. D'ora in poi solo Compendio (vedi in fine la descrizione).

I 8. Cito dal Compendio di cose nuove di Vincenzo Calmeta e altri auctori, Venezia, Zoppino I 507 (vedi oltre).

19. Carlo Delcorno, La predicazione nell'età comunale, Sansoni, Firenze i 974, p. I 8. La tecnica (vicina a quella utilizzata da Petrarca del De otio religioso) è portata all'estremo da alcuni predicatori, come Roberto Caracciolo, in realizzazioni virtuosistiche, ma facili oggetti di scherno. Cfr. ad esempio la descrizione di una predica in Folengo: «Durarat grossam iam praedica Cingaris horam, / quem cuncti fratrem pensassent esse Robertum: / allegabat enim Sextum, Decretale, Decretum, / Angelicam, Glosam, Bibiam, Sanctumque Tomasum» (Teofilo Folengo, Baldus, a cura di Emilio Faccioli, Einaudi, Torino i 989, IX 242$45)$.

20. Bernardino da Siena, Prediche volgari sul Campo di Siena. I427, a cura di Carlo Delcorno, Rusconi, Milano 1989 .

2 I. Sulla frottola cfr. Sabine Verhulst, La frottola (XIV-XV sec.): aspetti della codificazione e proposte esegetiche, Rijksuniversiteit Gent, Gent I990; Alessandro Pancheri, "Col suon chioccio». Per una frottola 'dispersa' attribuibile a Francesco Petrarca, Antenore, Padova I993; Marco Berisso, Che cos'è e come si dovrebbe pubblicare una frottola?, in «Studi di filologia italiana», LVII (I 999), pp. 20I-33. Per la zingaresca Franca Magnani, La zingaresca. Storia e testi di una forma, Zara, Parma i 988 .

22. Verhulst, La frottola cit., p. 52.

23. Berisso, Che cos'è e come si dovrebbe pubblicare una frottola? cit., p. 227.

24. Claudio Giunta, Sul rapporto tra prosa e poesia nel Medioevo e sulla frottola, in Storia della lingua e filologia. Per Alfredo Stussi nel suo sessantacinquesimo compleanno, a cura di Michelangelo Zaccarello e Lorenzo Tomasin, SISMEL Edizioni del Galluzzo, Firenze 2004, pp. 2 5-72, p. 48.

25. Cfr. ad esempio le indicazioni in tal senso di Jean-Claude Schmitt, Les traditions folkloriques dans la culture médiévale. Quelques réflexions de méthode, in «Archives de sciences sociales des religions», 52.I , (I98I), pp. 5-20. 


\section{Matteo Largaiolli}

26. Rouch, Diffusion orale cit., p. 36 (e sgg.): «Deux circuits très nettement différenciés répètent la bi-partition de la diffusion orale, l'un court et "privé" concernant la seule classe dominante, l'autre large et ouvert s'adressant à tous les publics».

27. Antiche rime venete (XIV-XVI sec.), a cura di Marisa Milani, Esedra, Padova I997, pp. 237-94 (nel codice alle cc. I05V-I 2or); i mariąi erano già in Emilio Lovarini, Antichi testi di letteratura pavana, Romagnoli-Dell'Acqua, Bologna i 895 .

28. Lodovico Dolce, Dialogo nel quale di ragiona delle qualità, diversità e proprietà dei colori, In Venetia, Giovanni Battista Marchion Sessa, i 565, pp. 80v-8 Ir.

29. Maria Teresa Casella e Giovanni Pozzi, Una «predica d'amore» attribuita a Francesco Colonna, in Vestigia. Studi in onore di Giuseppe Billanovich, a cura di Rino Avesani, Mirella Ferrari, Tino Foffano, Giuseppe Frasso, Agostino Sottili, Edizioni di storia e letteratura, Roma 1984, pp. $159-80$.

30. Cfr. Angela Nuovo, Il commercio librario nell'Italia del Rinascimento, FrancoAngeli, Milano I 999 , p. 102.

3I. La predica può essere intesa metricamente anche come una variazione su una serie di endecasillabi frottolati (o sul gliommero), dove un verso ogni tre conta due settenari anziché un endecasillabo.

32. Antonio Rossi, Lirica volgare del primo Cinquecento. Alcune annotazioni, in Forme e vicende. Per Giovanni Pozæi, a cura di Ottavio Besomi, Giulia Gianella, Alessandro Martini, Guido Pedrojetta, Antenore, Padova I988, pp. I 23-57, p. I 39 colloca in una linea di tipo erotico la materia trattata tra gli altri (Cei, Altissimo, Olimpo) da «Rasiglia, in specie nella "Quarta pars" della Predicar); Nadia Cannata, Il canzoniere a stampa (I470-I530). Tradizione e fortuna di un genere fra storia del libro e letteratura, Bagatto Libri, Roma 2000, p. 3 I 5 (n. 8 I) nella descrizione del Compendio (Zoppino I 507) segnala la «Predica [di Marco Rosiglia]»; Rinaldo Rinaldi, Umanesimo e Rinascimento, in Storia della civiltà letteraria italiana, diretta da Giorgio Bàrberi Squarotti, vol. II/2, Utet, Torino I993, p. 968 fa riferimento alla Predica del Compendio, e pur non avanzando un'attribuzione esplicita, collega il testo all'edizione delle rime di Rosiglia.

33. Ludovico Iacobilli, Biliotheca Umbriae sive de scriptoribus Provincial Umbriae Alphabetico Ordine digesta. Una cum Discursu praefatae Provinciae. Volumen primum, Fulginiae, Apud Augustinum Alterium, 1658, p. 195.

34. Michele Faloci Pulignani, Le arti e le lettere alla corte dei Trinci, in «Giornale storico della letteratura italiana», II (1883), pp. 28-58, pp. 55-57: «Aggiungo un poeta [...] nella lingua italiana assai valente. Marco da Rasiglia, che tale è il suo nome, fu autore di versi di amore, allora e poi stimati assai. Cantò egli la storia della Maddalena: compose strambotti, sonetti, ballate, canzoni, poesie popolari, parte edite, parte no, tutte ricercatissime, ma oggi assai difficili a ritrovare nelle rare stampe di quel tempo, che appena si mostrano in commercio, sono inesorabilmente portate via da bibliofili denarosis. Cfr. anche Michele Faloci Pulignani, I medici di Foligno e l'Università di Perugia, in «Bollettino della R. Deputazione di storia patria per l'Umbria» n. 53 (segnalato in «GSLI» LXVI, I91 5 , p. 292).

35. Opera de Maestro Marcho Rosiglia da Fuligno, in Venetia, Nicolò Zopino e Vincentio compagno, I 2 I, c. A2r: «Ad illustrissimam atque excellentissimam Dominam D. Elisabetham Gonzagam Urbini Ducissimam, Marcus Rosilia S. P. D.». Sul capitolo in morte del duca cfr. Guido Vitaletti, Assonanze dantesche in una epistola in versi del secolo XVI, in «Il Nuovo giornale dantesco», II (I918). 
36. Angela Carella, Urbino e le Marche, in Letteratura italiana. Storia e geografia. II/ I, L'età moderna, Einaudi, Torino I988, pp. 473-520, p. 49 I.

37. Cfr. Carella, Urbino e le Marche cit., pp. 490-96.

38. Carella, Urbino e le Marche cit., p. 490.

39. Alcune indicazioni sulle opere di Rosiglia si possono leggere in due interventi di Luisa Palpacelli, Le rime profane di Marco Rasiglia da Foligno, in «Bollettino storico della città di Foligno», Is (I99I), pp. 297-305, e di Piero Lai, "Frottola de cento romiti de Maestro Marcho Rosiglia da Foligno», in «Bollettino storico della città di Foligno», I7 (I993), pp. 33-52. 
Per un Repertorio delle Prediche D'Amore

PREDICHE MANOSCRITTE

I. Predica d'Amore.

Venezia, Biblioteca Marciana, It. IX I I I (6538), cc. 3 Ir - 37v.

Miscellaneo; XV secolo, $2^{\circ}$ metà; mm. I63 × I I $3 ;$ I +97 + I carte.

bibliografia: descrizione in Andrea Comboni, Per l'edizione delle Rime di Antonio Cornazano, in «Studi di Filologia Italiana», XLV (1987), pp. Io I-49, p. I09, e in Elena Maria Duso, Appunti per l'edizione critica di Marco Piacentini, in «Studi di Filologia Italiana», LVI (1998), pp. 57-I 27, p. 73 , con la bibliografia pregressa.

In prosa.

Tema: Omnia vincit Amor, et nos cedamus Amori (VIRG., Buc. x 69).

In tre parti, con divisio del tema: «Et prima dove dice Omnia vincit amor, vederemo quale è l'habito de questo Amore et cum quale arme sia sì victorioso. Secundo, vederemo quanto e quale sia la sua forza. Tertio, vederemo la cagione per la quale dovemo a lui acostarse, concludendo el nostro thema et nos cedamus amorì.

c. 3 Ir: Questa sie sentenzia e parlare del facundissimo poeta Virgilio / c. $37 \mathrm{v}$ : e cossì facendo lui ne farà gloriosi in terra et in ciel ne darà la corona dela gloria. Amen. Finis.

\section{Predica d'Amore.}

Firenze, Biblioteca Nazionale, Magliabechiano VII I030.

Cartaceo; codice composito, di tre sezioni (prima e terza: XVI secolo); le prediche nella seconda sezione, XV secolo, alle cc. 46-I 20; fascicoli irregolari (III: sei fogli; IV: tre fogli; VIII: quattro fogli; altri fascicoli: dispari, con fogli aggiunti).

Bibliografia: Milani; Lovarini; Pozzi Casella; Vittorio Rossi, Le lettere di messer Andrea Calmo, cit., p. C nota I cita la disperata anonima di c. $97 \mathrm{v}$.

Contiene quattro prediche d'Amore in prosa:

2.I cc. 75 r-83v Predicha d'Amor

Tema: Quo me fixit Amor, quo me violentius usit, / Hoc melior facti vulneris auctor ero (Ov., Ars am. I 23-24).

In tre parti, con distinctio di "Amore".

c. 75 r: Dexideroxo dela salute di zaschaduna persona / c. 83v: priego la madre Venere che ziaschaduna de voi presto conducha per infinita sechula sechulorum. Amen. Non iè salus...

Bibliografia: pubblicata da Maria Teresa Casella e Giovanni Pozzi, Una «predica d'amore» attribuita a Francesco Colonna, in Vestigia. Studi in onore di Giuseppe Billanovich, a cura di Rino Avesani, Mirella Ferrari, Tino Foffano, Giuseppe Frasso, Agostino Sottili, Edizioni di storia e letteratura, Roma 1984, pp. I59-80 (il testo alle pagine I65-80). I mariazi delle cc. 105 $\mathrm{V}$ - 
I $20 r$ in Antiche rime venete (XIV-XVI sec.), a cura di Marisa Milani, Esedra, Padova 1997, pp. 237-294; e già in Emilio Lovarini, Antichi testi di letteratura pavana, Romagnoli-Dell'Acqua, Bologna i 895 .

2.2 cc. $82 \mathrm{r}-85$ v Predica de pelegrinis

Senza tema.

In tre parti, con distinctio di "Amore".

c. $82 \mathrm{v}$ : Divideremo adoncha questa nostra predicha in 3 parte. Sula prima parte [parte in interlinea] vederemo che chosa hè amor. In la segonda parte [parte in interlinea, altro inchiostro] da che chosa nase et in che parte de la umana natura abia el suo locho. In la terza et ultima parte [c.s.] a che modo s'aquista e se chonserva.

c. 82r: Ritrovandomi zià molti zorni pasati nel dixerto hozioxo e senza alchun pensiere / 85 v: vui solo volete eser diforme da quelo che Dio, li zieli e la natura à ordinato? Finis.

2.3 cc. $85 \mathrm{v}-87 \mathrm{v}$ Predica di Zan de Chineto bereter in Marzaria

Tema: Diligite fratres uestros ut saluemini.

Divisa in un Sermo (in due parti) e in un Exenplo (da Valerius Maximus, Factorum et dictorum memorabilium libri 4.7.ext.I)

c. $85 \mathrm{v}$ : E mai ne la mia mente se muovase alchuna viturbazione / c. $87 \mathrm{v}$ : se questo farete, in questo mondo arete la grazia et in l'altro la santa gloria. Amen. Dio ve benediga.

2.4. cc. IOI $\mathrm{r}-\mathrm{IO} 2 \mathrm{~V}$ De peregrinis amoris

Predica incompleta.

Tema: Vere prius uolucres (Ov., Ars am. I, 27 I-274).

Divisa in tre parti, secondo quanto dichiarato nella Partium declaratio. c. Iorv: «divideremo adoncha la nostra predicha in tre parte. In la prima, averemo assapere chome ali gioveni si chonviene amare; nela sechonda, chomo la dona pare el più chrudele animalio in vista che sia chreato in terra; nella terza et ultima, chomo nesuno perfeto amore pò esere se non fra dui».

La predica si interrompe già nella prima parte, su una citazione di Tibullo.

c. Io Ir: Amantisime persone et dilectisimi mei, nui siamo peregrini sotoposti ala religione del dio d'amore / c. IO2v: ve chonverà fare chomo dize Tulio: Vidi ego qui iuvenum miseros lusiset amores / Post Veneris vinchulis subdere cholla senem. / Et sibi blandizias tremula chonponere voze / Et manibus crinas fingere vello chomas.

\section{Predica de Amor}

Sevilla, Biblioteca Colombina, 7-I-35.

Cartaceo, XV secolo, $2^{\circ}$ metà; $\mathrm{I} 2$ cc., 2 Io $\times$ I $52 \mathrm{~mm}$.

Scrittura corsiva, in un'unica mano (della stessa mano le correzioni e le aggiunte in interlinea). Il codice è oggi legato a una Comedia del amor di Ponžio di Caio Calorzo Ponzio. Gli angoli superiori e alcuni margini inferiori sono rovinati da macchie di umidità, $\mathrm{e}$ in più punti l'inchiostro è svanito e la lettura è difficoltosa. 
Bibliografia: nota sul codice in Carmen Álvarez Marquez, Catálogo de los manuscritos en italiano de don Hernando Colón (Biblioteca Capitulary Colombina de Sevilla), in Tra Siviglia e Genova: notaio, documento e commercio nell' età colombiana. Atti del convegno internazionale di studi storici per le celebrazioni colombiane (Genova, I 2-I4 marzo I992), a cura di Vito Piergiovanni, Giuffrè, Milano, pp. 229-326, p. 28 5-86.

In versi: endecasillabi frottolati (rimalmezzo oscillante tra settima e quinta sede); Oratio in quartine di ottonari (abab). Citazioni latine inserite in modo indipendente dalla scansione metrica.

Tema: Concordia parve res crescunt, discordia maxime dilabuntur (SALL., Bellum Iugurtbinum го, 6)

In tre parti, sui motivi di concordia, discordia, frutti della concordia.

c. Ir: Per impetrar la gratia di quella / i 2r: et in memoria / in l'altro havrai la gloria / per infinita secula seculorum. Amen. Laus finis deo

\section{Predicha d'Amore}

Venezia, Biblioteca Marciana, It. IX 3 I 0 (6650), cc. 44v-50v.

Cartaceo; XVI secolo, $2^{\circ}$ metà; 70 carte.

Scrittura corsiva; ex- libris «da Morelli Giov. (397)». 9 carte iniziali bianche; a c. Ior titolo "Libro di diverse compositione così volgari, come latine composte per diversi ingegni".

I testi contenuti sono piuttosto eterogenei: poesie a carattere religioso, encomiastico, amoroso, ma anche canti carnascialeschi e una frottola in settenari baciati di Belisario da Cingoli, Chi intende stia attento (cit. in Le lettere di messer Andrea Calmo, con introduzione ed illustrazioni di Vittorio Rossi, Loescher, Torino i 888, p. 247, n. 8). Tra gli autori compaiono Luigi Alamanni, il Lasca (Pover'buomini siamo oggi condotti), Torquato Tasso, con quattro madrigali, alle cc. 66v-67r: Al vostro dolce az₹urro (n. 6I 4, ed. Maier), Al tuo dolce pallore (n. 6 I s: «Al tuo vago pallore»), La bella pargoletta (n. 61 I), Caro amoroso neo (n. 602).

In versi; frottola di settenari a rima baciata, con ottava (Oratione, Spirito maligno che l'oscure grotte).

In tre parti; exempla da Boccaccio, Decameron VIII 7 e Decameron III Io.

c. 44v: Già rilucente e bella / c. 5ov: San Cresci-in-man tutte vi benedica.

PREDICHE A STAMPA

I. Filippo Baldacchini, Nox illuminata

In prosa.

Tema: 3 distici elegiaci che si rifanno alla tradizione elegiaca e virgiliana.

Verus amor dux est solus, magnusque potensque:

sydera vincit amor, tartara vincit amor.

Foelix qui grato servus bene servit amori:

aspera vincit amor, mollia vincit amor.

Rusticus est (moneo) siquis non novit amorem:

omnia vincit amor, singula vincit amor.

Bibliografia: su Baldacchini: Girolamo Mancini, Contributo dei cortonesi alla coltura italiana, R. Deputazione Toscana di Storia patria, Firenze 1922 ( ${ }^{a}$ edizione); G. Guglielmi, voce Baldacchini, Filippo, in Dizionario Biografico degli Italiani, Istituto dell'Enciclopedia Italiana, 
Roma I963. Cenni in Ignazio Baldelli, Correzioni cinquecentesche a Lorenzo Spirito, in «Studi di Filologia Italiana», IX (I95 I), pp. 39-I 22 (pp. 54-56).

La predica è nota in due redazioni diverse. La redazione più completa, con la ritrattazione della Predica, è:

a. Filippo Baldacchini, Nox illuminata

Città del Vaticano, Biblioteca Apostolica Vaticana, Capponi vi 5 I.

In $8^{\circ}$; fascicoli segnati (da $\mathrm{A}_{2}$ a $\mathrm{N}$ ), per carte in tutto; caratteri romani; stampa di Bartolomeo Zanetti (su cui Dennis Everard Rhodes, L'attivita tipografica di Bartolommeo Zanetti a Firenze I5I4-I524, in Miscellanea di studi in onore di Clementina Rotondi, pp. 25-32).

A[I]r: Philipp. Baldachini Coritani Nox illuminata

$\mathrm{N}$ [2]r: Impressioni florentiss(imae) fluentiae datum .VII. kl. / Nouemb. .M.D.XIX. op(er)a et cura uigili / Bartholomaei Brixiensis.

cc. Arv-A2v: lettera di dedica a Silvio Passerini.

Silvio Passerino Coritano: in Christo pr(esbiter)i ac / Domino Reverendissimo: Sanctaeq(ue) / Ro(manae) Ecclesiae benemerenti Datario / sub Medices Divo Leone .X. / Opt(imo) Max(imo) Pont(ifice) S(alutem) suiq(ue) / co(m)mendatione(m) D(ominus) / Coritanus Bal / dachinus / Philip / pus.

inc.: Antique consuetudinis erat non improbatum institum; expl.: ex veteri Gregoriana Academia. Idibus Augusti. MDXV.

cc. A[3]r-D[4]r: Predica.

$\mathrm{A}[3] \mathrm{r}$ : Sermo piissimus habendus in liberi patris festivitatibus toto orbe celebrari consuetis ad amentes et rudes ut amantes et sapientes fiant.

$\mathrm{D}[4] \mathrm{r}$ : per infinita secula seculorum. amen. Il fine.

cc. D[4]v-E2v: Indice del contenuto della Correctio

D[4]v: Adamiani .lxxxviii.; E2v: Vxor .xxiii.

cc. E[3]r-N[2]r: Correctio (ritrattazione della Predica)

$\mathrm{E}$ [3]r: Correctio predicatio- / nis sub titulo amo- / ris facte. Tema

$\mathrm{N}[2] \mathrm{r}$ : $\operatorname{co}(\mathrm{n})$ uoi $\mathrm{co}(\mathrm{n})$ duchi $\mathrm{p}(\mathrm{er})$ infinita secula seculor(um)

c. $\mathrm{N}[2] \mathrm{r}$ : Impressor Lectoribus [errata corrige]

inc.: In Enomao Sophocles pii lectores

Una redazione in parte diversa è testimoniata da:

b. Predica d'amore bellissima composta per el digno poeta Baldoino Cortonense.

Venezia, Biblioteca Nazionale Marciana, Miscell. I946, I4.

In $8^{\circ}$; 3 fascicoli segnati $\mathrm{A}_{\mathrm{I}}-\mathrm{C}_{4}$, per $\mathrm{I}_{2}$ carte in tutto; caratteri romani; titolo a caratteri gotici; la predica termina a c. $\mathrm{C}_{4} \mathrm{r}$ (c. $\mathrm{C}_{4} \mathrm{v}$ bianca).

BIBLIOGRAFIA: Max Sander, Le livre à figures italien: depuis I467 jusqu'à I530: essai de sa bibliographie et de son histoire, Hoepli, Milano 1942, n 4081: Madalius, Io. Bap. (Baldoino Cortonense). Predica d'amore. S.l.a. \& n.t. Contiene solo la predica.

Testimone non datato.

Nel frontespizio: stampa di donna seduta che incorona e porge una freccia a Cupido alato, bendato e armato di arco e faretra. La stessa stampa compare nel frontespizio dei Triumphi della felice memoria del preclarissimo poeta musico 
Vincenzo Calmeta. Opera molto dilectevole et novamente impressa, stampato, come da colophon, in Pesaro per Pietro Capha a instanzia di Nicolo Zopino: anche la stampa di Calmeta non ha date, ma la moderna editrice la colloca tra i 508 e I IO-I II (cfr. Calmeta, Triumphi, p. L; riproduzione del frontespizio tra p. XXVII e p. XXVIII).

A2r: ๆIO. BAP. MADALIVS COR / TONENSIS AD AMANTES [il gruppo di caratteri COR è stampato al rovescio]; [8 distici elegiaci; inc.:] Colla cupidineo subdis quicunq(ue) furori; $\mathbb{q B a l d u i n i}$ Cortonensis sermo deuotissimus in carnis preuio toto orbe celebrato die habe(n)dus ad exp(er)giscendos ame(n)tes ut aman(n)tes fia(n)t; Thema [3 distici elegiaci; inc.:] Verus amor dux est solus: magnusq(ue) potens$\mathrm{q}(\mathrm{ue})$; $\uparrow$ Sunt hæc uerba a cupidinis doctoribus fidedignis dicta \& i(n) n(ost)ræ (con)cionis redacta $\mathrm{p}(\mathrm{ro})$ positu(m); A2v: ACioche alcuna cosa alli naturali ingegni; $\uparrow$ Oratio ad Venerem cypridam. Venere sacra in ciel diuina stella; Thematis replicatio. Verus amor dux est solus. Et reliqua; Benche moltissimi excelsi \& potenti dei \& dee; Bıv: Pars Prima. $\mid$ Homo non est qui amorem nescit. Si uero homo ex parte natura rudis est. Di quanto Fructo \& excellentia sia; B4r: Pars seconda. Pamphilo Saxo, Quicquid agu(n)t sup(er)i iustu(m) est: non sydera possunt / Criminis iniusti turpia signa pati. PErche credete uoi o mente stolte; $\mathrm{C}_{3}$ r: Pars tertia postremaq(ue). Labitur citatis tempus cursibus. Tanto piu la natura capiente desiderosa fare si suole; $\mathrm{C}_{4} \mathrm{v}$ : Ilche face(n)do in questa uita mortale haueriti el summo dilecto et piacere \& nellaltra la gloria per infinita secula seculor(um). Amen.

Altre copie:

Folger Library, Washington [234-030q].

Non ho controllato personalmente la copia, ma dalla descrizione catalografica si apprende che a carta A2r il gruppo «COR» della parola "Cortonensis" «is printed upside down», come nell'esemplare della Biblioteca Marciana.

2. Francesco de' Canti, Predica d'Amore, Paulo Danza [Venezia].

[Piacenza, Biblioteca Passerini Landi]

Predica D'Amore edita per lo Eccellentissimo Dottore delle arte et medicina messer Francesco de Canti detto Raynaldo Mantuano.

In prosa.

Tema: Quid vos perdiderit dicam nescitis amare. / Ovidii tertio Artis Amandi (Ov., Ars am. III, 4I).

In $8^{\circ}$; caratteri romani; 8 carte segnate Aii (Ai è il frontespizio), B, Bii. In frontespizio, oltre alla titolazione, compare stampa di un predicatore (?), coronato di alloro, seduto in cattedra, con libro aperto e penna nella mano destra; in basso, tre scrivani (sotto il dotto in cattedra, alla sua destra e alla sua sinistra), con libro e penna.

\section{Contiene:}

I. (Predica) Av: [Thema] TQuid uos perdiderit dicam nescitis amare. Ouidii terzio Artis Amandi; LI orrendi \& spauentosi precipitii; Aiiv: Prima Parte. HOr 
pigliamo la prima parte; $\mathrm{Bv}$ [Seconda parte] NElla seconda parte habbiamo da uedere como il se debbe amare; $\mathrm{B}_{3}$ r: in questo mondo starete in letitia: \& in laltro Ioue faccia quello habbia essere \& cetera. FINIS.

2. B 3 r: [dp]Capitolo posto in canto. DVra passion che per amor soporto; B 3 v: Non uedendo il bel uolto che me ha morto. FINIS.

3. B3v: [4 strambotti dialogati] GLie pur cocente il fier desir che ho in core; B4r: Così se uince un cor pregando e amando. FINIS.

B4r: Per Paulo Danza.

Altra edizione:

Francesco de Canti, Predica de amore. Cosa noua. / Predica / De Amore / Edita per lo excellentissimo doctore de le arte / \& medicina Miser / Francisco de canti / ditto Rainaldo / Mantuano

Roma, Bertocho stampatore [1 $52-]$

$8 \mathrm{cc}$, in $8^{\circ}$.

Cambridge, Library of Cambridge University [Fi s 2.e.2.I I]

BibLiografia: Victor Scholderer, A Sixteenth-century Bertocbus, in Contributi alla storia del libro italiano. Miscellanea in onore di Lamberto Donati, Firenze, Olschki 1969, pp. 297-304.

Francesco de' Canti, o Raynaldo Mantovano, è ricordato anche come autore di facezie: Facecie del Gonella composte per maestro Francesco dicto maestro Raynaldo da Mantua, in Bologna, per Justiniano da Rubiera, I 506 a dì ultimo de Aprile. (2 cc., in $4^{\circ}$ ); e di un'Opera nova amorosa stampata a Venezia, ad istantia del maestro Rainaldo Mantuano (databile al is 25 circa), conservata nella collezione della Fondation Barbier-Mueller a Ginevra.

3. Pier Luigi di Francesco Magdoli, Due prediche d'Amore carnascialesche, Michelangelo de' Libri, Siena i 524.

[London, The British Library, C. I92. a. 2 I 5 ]

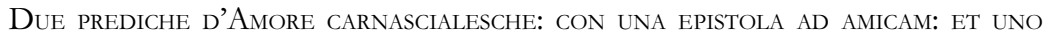
CAPitolo. Composto per Pier Luigi di Francesco Magdoli da Colle di Valdelsa.

Stampato in Siena. Adì xxv di Giugno: per Michelangelo di Bart. F. Ad instantia di Maestro Giovanni di Alixandro Libraro. Nel i 524.

In versi: frottole sul metro della zingaresca: $a b(b) C, c d(d) E$, con molti versi ipometri o ipermetri.

In $8^{\circ} ; 8$ cc., segnate $A-B_{2}$; caratteri romani. In fine: marca con le iniziali $G($ iovanni) $\mathrm{L}$ (andi).

Bibliografia: descritta in Sander, Le livre cit., n. 4 I I 5 , fig. 709.

Il cambio di parte è segnalato con un segno di paragrafo a inizio parte (e un punto in basso a fine). Contiene:

I. Av: Predica Prima Carnascialesca; [due ottave:] CHe ualse ad Cicero(n) ta(n)ta eloque(n)tia; Regina celi in ciel su nostra Venere; A2r: IN declaratione / materie presentis / oportet audientis / stare attento; A4v: Du(n) suono d'unaltro nerbo / 
chio so che piu ti piace / pero ualete impace / \& state sani. Finis. $\|$ Finita la Prima Predica.

2. A4v: qPredica Seconda. [Sonetto caudato:] PER non si deuiare dal consueto; qInuocatione. [ottava:] Madre de pescatori sancta pigrizia; Br: INanzi ad laurora / inuisione mapparue / \& poi subito sparue / un bel uechione; B2v: Et perche lhora nostra / io uegho trapassare / pero ti vo lassare / Valete in pace. FINIS. 3. B3r: "Epistola ad Amicam. SUggecto son con patientia amara; B3v: Perche uiuendo so che inuechierai. |FINIS.

4. B3v: Capitolo. QVal mercata(n)te che ha nel Mar sua barcha; B4r: Conosciuto post mortem buono amante. $\|$ FINIS.

5. B4v: Oratio [capitolo volgare - latino] Io so chio non son degno eterne deus; Quia pro nobis tu es homo factus. शFINIS.

B4v: Stampato in Siena. Adi .xxv. di Giugno: per Michelangelo di Bart. F. Ad instantia di Maestro Giovanni di Alixandro Libraro. Nel i 24.

Lo stampatore è Michelangelo de' Libri: anche la predica del Verde Lauro (qui n.

4) è «Stampata in Siena per Francesco Avanni e Giovanni di Alissandro Librari.

Adì 3i. di Agosto. MDXXXVI».

4. Verde Lauro, Predica de Amore Bellissima, Avanni, Siena i 536.

[Wolfenbüttel, Herzog August Bibliothek A: 107.22 Eth. (1 8)]

Predica de Amore Bellissima. Composta per el Verde Lauro.

B4v: Stampata in Siena per Francesco Avanni e Giovanni di Alissan dro Librari. Adì .3 I. di Agosto. M.D.XXXVI.

In versi: endecasillabi frottolati (con due ottave di introduzione)

Tema: Diliges proximum tuum sicut te ipsum (МАттн. 22, 39).

In $8^{\circ} ; 8$ carte; caratteri gotici; frontespizio con cornice floreale. Contiene solo la predica.

I. Arr: El tema; Inuocatio [ottava: Perche non si puo far cosa nel mo(n)do]; Salutatio [ottava: AVe terrestre e degna Imp(er)atrice]; Arv: Exordio (Carissimi doctores); Aiiv: Prima parte (Ho letto el gran Pittagora); A4v: Seconda parte (VOs omnes qui transitis); B $_{3}$ r: Terza (et) ultima Parte (Venite post me omnes amatores); $\mathrm{B} 4 \mathrm{~V}$ (hauer interra honor: in ciel la gloria). FINE.

Come già le prediche di Magdoli, anche la predica del Verde Lauro è stampata a Siena per diretto intervento di Giovanni di Alessandro Libraro (= Giovanni Landi) e Francesco Avanni. Nei repertori, l'attività editoriale di Avanni è sempre collegata a Giovanni Landi; Avanni continuò l'attività a Siena almeno fino al I 549 . L'origine in ambiente senese, oltre che dal luogo di stampa, è confermata dai ripetuti accenni espliciti alla città che si trovano nel testo.

Una predica è nota in due testimoni, a stampa e manoscritto:

a. Predica d'Amore novamente stampata.

[Wolfenbüttel, Herzog August Bibliothek M: Lk Sammelbd 64 (50)] 
Predica d'amore nouamente stampata.

In versi: frottola di settenari in quartine (abbc, cdde...)

Tema: Omnes humanos sanat medicina doloris / solus Amor morbi non amat artificem (Prop., El. II I, 57-58).

[ca. I 5 50]; 4 carte; con stampa: un predicatore a destra, sulla cattedra, che parla a un gruppo di sette giovani (due in primo piano seduti: un «sacerdote che predica ai suoi alunni» secondo Milchsack-D’Ancona); in $4^{\circ}$; caratteri romani.

Aır: Incipit salutatio (SAlue regina amoris); Aıv: qProemio (Per impetrar la gratia), TPrima pars (DIce Ouidio estima); A2v: Seconda pars (SEra adunque iocunda); A3v: Tertia pars (OGni siluestre quertia); A4v (Cercate tener cura / de tutti i vostri amanti / restati tutti quanti / Che amor vi benedica. FINIS).

c. A4v, dopo la Predica, una breve Epistola d'amore: Abenchè indegno sia unica signora mia [...] et da vostra candida mano aspetto dolce et suave risposta. Vale.

BIBLIOGRAFIA: la stampa è descritta da Milchsack-D'Ancona e da Sander, Le livre cit., n. 5863. Il primo recensore parla di quaranta strofe per parte, ma l'indicazione è imprecisa. Due farse del secolo $X V I$ riprodotte sulle antiche stampe con la descrizione ragionata del volume Miscellaneo della Biblioteca di Wolfenbüttel contenente Poemetti popolari italiani compilata dal Dott. G. Milchsack con aggiunte di A. D’Ancona, G. Romagnoli, Bologna 1882 (rist. anast. Forni, Bologna i 968), pp. 2 I4-I 5 (L. Predica d'Amore).

Francesco Novati, Tre lettere giocose di Cecco d'Ascoli, in «Giornale Storico della Letteratura Italiana», I (I 883), pp. 62-74; Erhard Lommatzsch, Beiträge zur älteren italienischen Volksdichtung. Untersucbung und Texte, Band I: Die Wolfenbütteler Sammelbände, Akademie-Verlag, Berlin I950, p. 5; p. I6.

Piero Camporesi, La maschera di Bertoldo. G. C. Croce e la letteratura carnevalesca, Einaudi, Torino 1976 , pp. $171-72$.

\section{b. Mantova, Biblioteca Comunale, A. I. 4}

Cartaceo, miscellaneo, inizio XVI secolo; $152 \times 108 \mathrm{~mm}$; ro carte numerate con tavola dei componimenti; 264 carte con numerazione originale; 2 carte iniziali non numerate (aggiunta posteriore). Il codice è databile ai primi anni del XVI secolo: contiene rime in morte di Serafino Aquilano - che è anche l'autore più rappresentato. Contiene 374 testi, in gran parte di tradizione musicale ( 72 sonetti, I 28 strambotti, 45 ode e canzonette, 83 frottole, 37 barzellette, 8 capitoli, I canzone), dal «dettato fra aulico e popolaresco» (Gallico, Un libro, p. 55).

La predica d'Amore alle cc. $237 \mathrm{r}-253 \mathrm{v}$.

Bibliografia: il manoscritto è stato studiato da Claudio Gallico, Un libro di poesie per musica dell'epoca d'Isabella d'Este, Bollettino Storico Mantovano, Mantova I96I (tavola alle pp. 7 II०8).

La predica, nella redazione del manoscritto, presenta due ampie interpolazioni dalla Predica di Rosiglia. Manca l'indicazione del thema. 
Matteo Largaiolli

La PRedica attribuita a Marco Rosiglia

TESTIMONI MANOSCRITTI

Modena, Biblioteca Estense Universitaria, codice Campori i 87 ( $\gamma$.F.6.1 5 ).

Cartaceo; inizi XVI secolo; mm. 207xi 35; 89 carte.

La Predica è alle carte $28 \mathrm{r}-35 \mathrm{v}$, adespota e mutila (mancano Thema e Oratio ad Venerem; inizia con il Proemio).

La datazione non è certa, ma il codice contiene le Stanze di Bembo «in una redazione anteriore a quella comparsa a stampa» (Leonardi, p. 4); vi si legge anche il capitolo di Ariosto O più che 'I giorno a me lucida et chiara; il codice condivide inoltre alcuni testi con il Compendio de cose nove I 507: Sacri sepulcri, santi mausolei (c. 36v), Sochorri caro amico al nuovo spasimo (c. 40v), Chi pensa a questa vita miserabile (c. 48r), Per segno del mio amor nel fronte porto (c. $52 \mathrm{~V}$ ).

BiBLIOGRAFia: Laura Leonardi, Il codice Campori 187 , in «La Bibliofilia», 85 (1983), pp. 3-25. Pietro Bembo, Stanze, edizione critica a cura di Alessandro Gnocchi, Società editrice fiorentina, Firenze, 2003, pp. XxxvIII-XL.

TESTIMONI A STAMPA

I. Compendio de cose nove di Vicenzo Calmeta \& altri auctori cioe Sonetti Capitoli Epistole Egloghe pastorale Strambotti Barzelette \& una Predica damore.

Venezia, Nicolò Zoppino, i 8 luglio I 507

[London, British Library, G. Io646]

Compendio de cose noue di Vice(n)zo [sic] Cal / meta \& altri auctori cioe Sonetti Capitoli / Epistole Egloghe pastorale Stra $(\mathrm{m})$ botti Bar $=/$ zelette $\&$ una Predica damore.

$\mathrm{M}_{3} \mathrm{v}$ : Stampato in Venetia per Nicolo / dicto Zopino nel anno del no= / stro signore. M.CCCCC:VII / Adi .XVIII. de Luio.

In $8^{\circ}$, carte segnate $\mathrm{A}-\mathrm{M}_{4}$; la Predica, adespota, alle cc. $\mathrm{K}_{3} \mathrm{v}-\mathrm{M}_{3} \mathrm{v}$. Nel frontespizio una xilografia di un uomo che suona la lira e Cupido che lo mira con una freccia.

BIBLIOGRAFIA: Nadia Cannata, Il canzoniere a stampa (I470-I530). Tradizione e fortuna di un genere fra storia del libro e letteratura, Bagatto Libri, Roma 2000 (n. 8I); Marisa Milani, Sonetti ferraresi del '400 in una raccolta di poeti cortigiani, in "Giornale storico della letteratura italiana», I 50 (1973), pp. 292-322; Letizia Mazzella, Per un'edizione delle rime di V. Calmeta, Adriatica Editrice Salentina, Lecce 1981. Antonio Rossi, Opera noua composta per diuersi auctori. Un'antologia del I502, in Il libro di poesia dal copista al tipografo, a cura di Marco Santagata e Amedeo Quondam (Ferrara, 29-3 I maggio 1987), Panini, Modena 1989, pp. I 57-76. Antonio Rossi, Lirica volgare del primo Cinquecento. Alcune annotazioni, in Forme e vicende. Per Giovanni Pozzi, a cura di Ottavio Besomi, Giulia Gianella, Alessandro Martini, Guido Pedrojetta, Antenore, Padova I988, pp. $123-57$. 
Altre edizioni del Compendio de cose nove.

2. Compendio de cose noue de Vicenzo Calmeta \& altri auctori cioe sonetti capitoli epistole egloghe pastorale strambotti barzelette et una predica damore.

In Venetia, per Manfredo de Monferrato, 8 luglio I 508

[Roma, Biblioteca Angelica, R R 3 i 7 ]

3. Compendio de cose nobile et delectevole de Vincenzio Calmeta et D'altri auctori: cioe Sonetti, Capitoli, Epistole, Egloge pastorale, Strambotti, \& Barzellette con una Predica d'Amore.

Venezia, Simon de Luere.

[citato da Francesco Saverio Quadrio, Della storia, e della ragione d'ogni poesia, Francesco Agnelli, Milano 174I, vol. II, p. 217, che lo ritiene ristampa di Opera nuoua, Rusconi I 507; Cannata, stc. I 53 ].

4. Compendio de cose nuoue di Vincenzo Calmeta altri auctori cioe sonetti capitoli epistole egloghe pastorale strambotti barzellette \& vna predica damore.

In Venetia, per Melchior Sessa, i 5 i 5 .

[Firenze, Biblioteca Nazionale, E.6.6.89.]

5. Compendio de cose noue Vicenzo Calmeta \& altri auctori cioe: sonetti capitoli epistole egloghe pastorale strambotti barzellete. Et una predica damore.

In Venetia, per Alexandro di Bindoni, 4 novembre i 5 i 5 .

[Modena, Biblioteca Estense Universitaria, $\alpha$ Z.7.I 5.$]$

6. Compendio de cose noue de Vincenzo Calmeta \&o altri auctori cioe sonetti capitoli epistole egloghe pastorale strambotti barzelette et una predica damore.

In Venetia, per Georgio de Ruschoni, 24 gennaio i 56

[Venezia, Bibioteca Nazionale Marciana, Misc. 2409.I.]

7. Compendio de cose noue Vincenzo Calmeta et altri auctori cioe sonetti capitoli epistole egloghe pastorale strambotti barzellete et una predica damore.

In Venetia, per Ioanne Tacuino de Tridino, i 5 I 7.

[Roma, Biblioteca dell'Accademia Nazionale dei Lincei e Corsiniana, I 3 I d 25 ]

8. Vincenzo Calmeta poeta vulgare: non manco facetto che elegante, Sonetti. Strambotti. Eccloghe. Capituli. Dialoghi. Et vna predica damore cosa bellissima.

In Chiuasso, per Francesco Garrone de Liuorno, i 29 del mese de luio.

[Pisa, Biblioteca universitaria].

STAMPE POPOLARI

I. Predica nouamente composta per uno divoto servo.

Venezia, Manfredo Bonelli de Monferrato, 8 luglio i 508.

[Siviglia, Bibl. Colombina I 4.I.9 (6)]

In $8^{\circ} ; 8$ carte segnate A-B4. A c. B4r la frottola Nacque al mondo per amare; $\mathrm{B}_{4} \mathrm{v}$ Mai ti voglio abandonare; Stampato in Venetia per Manfredo de Monteferrato ne l'anno del nostro signore MDVIII Adì VIII de luio. 
Bibliografia: cfr. Klaus Wagner-Manuel Carrera, Catalogo dei libri a stampa in lingua italiana della Biblioteca Colombina di Siviglia, Panini, Modena i 991 (n. 680).

2. Predica de Amore. S.l.a. \& N.t. (Venezia, c. I 5 I 3 - I 25 secondo Sander, Le livre cit., n. 5860$)$.

[Milano, Biblioteca Trivulziana. coll. H I I 3]

4 carte segnate A, Aii; caratteri romani, testo su due colonne (indico con a, b). Contiene la Predica e una frottola (stampata in caratteri gotici):

I. Ar a: Predica de Amore; OMnia vi(n)cit amor \& nos cadamus amori; Per impetrar gratie, dal diuin tribunale; Ar b: Oratio ad Venerem. [ottava:] VEnere radiante stella in cielo; Thematis repetitio. Omnia vi(n)cit amor \& nos cedamus amori; Av a: Loco superiori \& auctore. Proemio. DAl tenerello fiore de mie giouenil an(n)i; Av b: Prima parte. POtria farui al p(re)sente, auctorita molte; A 2r b: Seconda pars. ALcu(n) degno auctore, chamore ha diffiinito; $\mathrm{A}_{3} \mathrm{r}$ a: Tertia pars. DIco ch(e) riuolta(n)do, diversi texti \& iose; $A_{3} r$ b: Quarta pars. SE be(n) co(n)sideriamo, el fin diq(ue)sto amor(e); $A_{3} \mathrm{v}$ b: Alaq(ua)l co(n) uictoria, uos p(er)ducat dominus FINIS.

2. B4r a: [Frottola] BRigata sel vi piace / de star aldir in pace / Una opera molto bella / Che frotola sappella; B4r b: Viva il co(n)te farina chiamato da la gente / In q(ue)lla casa doue che no(n) e se fa gra(n) ste(n)te. Finis.

3. Predica damore. [Ser Piero Pacini da Pescia: Florence, i 5 I o?]

[London, British Library i I 42 r.b.i 4 ]

In $8[\mathrm{fb}] ; 4$ carte, caratteri romani. Il catalogo della British Library assegna la predica a Filippo Baldacchini, ma è la Predica che si legge anche nel Compendio.

4. Predica d'Amore molto piacevole e bella. Nuovamente ristampata, Firenze i 556.

[London, British Library i I 427.b.57]

In $8[\mathrm{fb}] .4$ carte. Come per l'altra stampa fiorentina, il catalogo della British

Library assegna la predica a Filippo Baldacchini, ma è la Predica che si legge anche nel Compendio.

5. Predica damore composta per un deuoto seruo del'amore. Intitulata omnia vincit amor In Chiuasso, per Francesco Garrone de Liuorno, i 29 del mese de zugno. In $8^{\circ} ; 8$ carte.

EDIZIONi di Marco Rosiglia

I. Opera del dignissimo doctore medico et poeta maestro Marcho Rosiglia da Fuligno, cioe Sonetti: Capituli: Egloghe: Strambotti: et due prediche damore.

In Venezia, Nicolò Zoppino, i 5 i 5 .

[B. Apostolica Vaticana Capponi VI i 37; Firenze, Biblioteca Nazionale Palat. D.4.7.67.I]

In $8^{\circ} ; 64$ carte segnate $A-Q_{4}$. La Predica alle cc. $\mathrm{I}_{2} \mathrm{v}-\mathrm{L}_{1} \mathrm{v}$ 
2. Opera noua del Preclarissimo poeta [...] Marcho Rosiglia [...] et altri auctori.

In Venezia, Giorgio Rusconi, Io gennaio i 5 I6.

[Milano, Braidense]

3. Oprera [sic] nona del Preclarissimo Poeta Mastro Marcho Rosiglia da Foligno et altri auctori. Nouamente stampata cioe Sonetti. Capituli Egloghe Strambotti. Una Predica damore. Et una frottola De cento romiti.

In Venezia, Giovanni Tacuino de Tridino, 28 ottobre i 5 I7.

[Venezia, Biblioteca Nazionale Marciana, Misc. 2432.6]

4. Opera de Maestro Marcho Rosiglia da Fuligno novamente corretta con aditione, Venezia, Nicolò Zopino e Vincenzo di Paolo, ig febbraio i 52 I.

[Bolzano, Biblioteca Civica Cesare Battisti, Rari A. 65]. 